\title{
ANÁLISIS DE LAS TASAS DE INTERÉS DE REFERENCIA EN COSTA RICA, EN MONEDA NACIONAL Y EXTRANJERA, DESDE UNA PERSPECTIVA DE INVERSIÓN FINANCIERA UTILIZANDO LA METODOLOGÍA DEL EFECTO FISCHER INTERNACIONAL
}

\author{
ANALYSIS OF THE REFERENCE INTEREST RATES IN COSTA RICA, IN NATIONAL AND \\ FOREIGN CURRENCY, FROM A FINANCIAL INVESTMENT PERSPECTIVE USING THE \\ FISCHER INTERNATIONAL EFFECT METHODOLOGY
}

ANÁLISE DAS TAXAS DE JURO DE REFERÊNCIA NA COSTA RICA, EM MOEDA NACIONAL E ESTRANGEIRA, A PARTIR DE UMA PERSPECTIVA DE INVESTIMENTO FINANCEIRO USANDO A METODOLOGIA DO EFEITO FISHER

\section{Carlos Leonardo Arguedas Campos ${ }^{1}$ Gustavo Mauricio Vallejo Esquivel ${ }^{2}$}

Resumen

El propósito del presente artículo es analizar tres indicadores del Banco Central de Costa Rica (BCCR): la tasa básica pasiva (TBP), el tipo de cambio del colón con respeto al dólar estadounidense (TC) y la tasa efectiva en dólares (TED); todos estos como componentes macroeconómicos que afectan la valoración de una inversión en colones o en dólares. El estudio se hará mediante la construcción de un modelo del efecto Fischer internacional (EFI), para el período comprendido entre el primer trimestre de 2016 y el primer trimestre de 2019. A partir de dicho modelo se evaluará, si durante este período invertir en dólares puede generar una ganancia de capital, y se esbozará la importancia que tienen los cambios absolutos del tipo de cambio para realizar dichas inversiones.

Doi: https://doi.org/10.15359/eys.24-56.2

Fecha de recepción: 29-05-2019. Fechas de reenvíos: 30-07-2019, 26-08-2019. Aceptado el 29-08-2019. Publicado el 30-08-2019.

${ }^{1}$ Economista. Máster en Política Económica. Académico de la Escuela de Economía, Universidad Nacional, Costa Rica. Correo electrónico: cargueda@una.ac.cr ORCID: https://orcid.org/0000-0002-4201-8263

${ }^{2}$ Máster en Banca y Finanzas. Máster en Negocios Internacionales. Académico de la Escuela de Administración de la Universidad Nacional, Costa Rica. Correo electrónico: gustavo.vallejo.esquivel@una.ac.cr ORCID: https://orcid.org/0000-0002-5809-9205

Carlos Leonardo Arguedas Campos y Gustavo Vallejo Esquivel 
Palabras clave: ganancia de capital; decisión financiera; crédito; tipo de cambio observado; indicadores económicos.

\begin{abstract}
The purpose of this article is to analyze, three indicators of the Central Bank of Costa Rica (BCCR): the basic passive rate (TBP), the exchange rate of the colon with respect to the US dollar (TC) and the effective rate in dollars (TED); as macroeconomic components that affect the valuation of an investment in colones or dollars. The study is carried out through the construction of an International Fischer Effect model (EFI), for the period between the first quarter of 2016 and the first quarter of 2019. Based on this model, it is evaluated, if during this period investing in dollars can generate a capital gain; in addition, the importance of absolute exchange rate changes to make such investments in dollars is outlined.
\end{abstract}

Keywords: capital gain; financial decision; credit; observed exchange rate; economic indicators.

\title{
Resumo
}

O objetivo deste artigo é analisar três indicadores do Banco Central da Costa Rica (BCCR): a taxa passiva básica (TBP), a taxa de câmbio do cólon em relação ao dólar americano (TC) e a taxa efetiva do dólar (TED) ; tudo isso como componentes macroeconômicos que afetam a avaliação de um investimento em cólon ou dólar. O estudo será realizado através da construção de um modelo internacional de efeito Fischer (EFI), para o período entre o primeiro trimestre de 2016 e o primeiro trimestre de 2019. Com base nesse modelo será avaliado se, durante esse período, investir em dólares pode gerar um ganho de capital e será delineada a importância de mudanças absolutas na taxa de câmbio para realizar esses investimentos.

Palavras-chaves: ganho de capital; decisão financeira; crédito; tipo de cambio observado; indicadores econômicos.

\section{Introducción}

En Costa Rica, en la primera mitad del siglo XVIII, se utilizaron diferentes medios de pago para el intercambio de mercancías, entre ellos, el tabaco y el cacao; pero no será sino hasta la segunda administración del jefe de Estado Braulio Carrillo Colima en 1839, cuando inicia la primera emisión 
del que se puede considerar el primer papel moneda costarricense, nacido conjuntamente con las primeras políticas monetarias del país, enfocadas en desarrollar un recién modelo agroexportador, impulsado y predominado por el café.

En 1914, en su discurso ante el Congreso Constitucional, el expresidente de la República Alfredo González Flores retomó la idea de cuando fuera diputado en el periodo 1910-1914, de que durante su administración impulsaría la creación de un banco hipotecario con el objetivo de que dicha institución coadyuvara con la producción nacional. Logró, de esta manera, que el 9 de noviembre de 1914 se constituyera el Banco Internacional de Costa Rica, ahora sí, con el fin expreso de desarrollar la actividad agrícola y rural del país.

La importancia de este banco radica en que en la década de los años treinta del siglo XX, cambiaría su nombre a Banco Nacional de Costa Rica y asumiría las funciones de emisor de la moneda costarricense hasta el año 1950, cuando se crea el Banco Central de Costa Rica (BCCR), institución rectora, hasta el día de hoy, en materia monetaria del país.

La evolución del sistema financiero nacional desde el año 1863, con la creación del Banco Anglo Costarricense, al año 2017, con el cierre de Bancrédito, se muestra en la Tabla 1.

Tabla 1

Síntesis de la cronología del sistema financiero costarricense

\begin{tabular}{|c|l|c|l|}
\hline Año & \multicolumn{1}{|c|}{ Evento } & Año & \multicolumn{1}{|c|}{ Evento } \\
\hline 1863 & Banco Anglo Costarricense & 1994 & Cierre del Banco Anglo Costarricense \\
\hline 1877 & Banco de la Unión & 1995 & $\begin{array}{l}\text { Ruptura del monopolio de las cuentas } \\
\text { corrientes y redescuento }\end{array}$ \\
\hline 1890 & $\begin{array}{l}\text { Banco de la Unión se transforma en } \\
\text { actual Banco de Costa Rica }\end{array}$ & 1995 & Nace la SUGEF \\
\hline 1914 & Banco Internacional de Costa Rica & 1998 & Crisis financiera del movimiento cooperativo \\
\hline 1918 & Banco Crédito Agrícola de Cartago & 2004 & Cierre de operaciones de BICSA \\
\hline 1945 & Banco Central de Costa Rica & 2008 & Crisis económica mundial \\
\hline 1948 & $\begin{array}{l}\text { Se decreta la nacionalización } \\
\text { bancaria }\end{array}$ & 2016 & $\begin{array}{l}\text { Situación de financiamiento de cemento } \\
\text { chino banca pública y privada }\end{array}$ \\
\hline 1976 & BICSA & 2017 & Cierre de Bancrédito \\
\hline 1987 & $\begin{array}{l}\text { Crisis del sistema financiero } \\
\text { informal }\end{array}$ & \multicolumn{2}{|l}{} \\
\hline
\end{tabular}

Fuente: elaboración propia con información de SUGEF.

Como se aprecia en la Tabla 1, el modelo bancario costarricense ha venido evolucionando por casi dos siglos hasta alcanzar la complejidad y desarrollo con que cuenta hoy en día el sistema 
financiero nacional y las políticas económicas emanadas por el BCCR y su Ley N ${ }^{\circ} 7558$ del 03 de noviembre de 1995, que reza en sus artículos del 1 al 3 lo siguiente:

Artículo 1. Definición

El Banco Central de Costa Rica es una institución autónoma de derecho público, con personalidad jurídica y patrimonio propios, que forma parte del Sistema

Artículo 2. Objetivos

El Banco Central de Costa Rica tendrá como principales objetivos, mantener la estabilidad interna y externa de la moneda nacional y asegurar su conversión a otras monedas y, como objetivos subsidiarios, los siguientes:

a) Promover el ordenado desarrollo de la economía costarricense, a fin de lograr la ocupación plena de los recursos productivos de la Nación, procurando evitar o moderar las tendencias inflacionistas o deflacionistas que puedan surgir en el mercado monetario y crediticio.

b) Velar por el buen uso de las reservas monetarias internacionales de la Nación para el logro de la estabilidad económica general.

c) Promover la eficiencia del sistema de pagos internos y externos y mantener su normal funcionamiento.

d) Promover un sistema de intermediación financiera estable, eficiente y competitivo.

Artículo 3. Funciones esenciales

Para el debido cumplimiento de sus fines, le competerán al Banco Central, de acuerdo con la ley, las siguientes funciones esenciales:

a) El mantenimiento del valor externo y de la conversión de la moneda nacional.

b) La custodia y la administración de las reservas monetarias internacionales de la Nación.

c) La definición y el manejo de la política monetaria y cambiaria.

d) La gestión como consejero y banco-cajero del Estado.

e) La promoción de condiciones favorables al robustecimiento, la liquidez, la solvencia y el buen funcionamiento del Sistema Financiero Nacional.

f) La emisión de billetes y monedas, de acuerdo con las necesidades reales de la economía nacional.

g) La determinación de políticas generales de crédito y la vigilancia y coordinación del Sistema Financiero Nacional.

h) La custodia de los encajes legales de los intermediarios financieros.

i) El establecimiento, la operación y la vigilancia de sistemas de compensación.

j) El establecimiento de las regulaciones para la creación, el funcionamiento y el control de las entidades financieras. 
a) La colaboración con los organismos de carácter económico del país, para el mejor logro de sus fines.

El desempeño de cualesquiera otras funciones que, de acuerdo con su condición esencial de Banco Central, le correspondan.

Dada la actual normativa, el permite a los grupos profesionales vinculados al área económica y financiera, dar constante seguimiento a los indicadores macroeconómicos imperantes en el país, para así desarrollar la capacidad de vislumbrar un panorama de su entorno y generar proyecciones y recomendaciones como herramientas de gestión empresarial y toma de decisiones para la disminución de riegos en las inversiones y la maximización de resultados económicos y financieros.

A partir de la última década, consecuencia del comportamiento que han presentado las tasas de interés ofrecidas por las instituciones financieras, tanto para créditos como para captaciones, se han generado cuestionamientos en los inversionistas en torno a qué posición asumir y qué recomendaciones existen en cuanto a endeudarse o invertir en una u otra moneda y el riesgo asociado a tales decisiones.

Lo anterior es aún más relevante cuando las instituciones financieras, junto con desarrolladores inmobiliarios, compiten en ferias como Expovivienda y Expomóvil para promocionar a los clientes potenciales productos diferenciados, mediante la publicidad de las mejores condiciones, beneficios y facilidades a su público usuario, aprovechando el comportamiento económico de las tasas de interés, lo que permite ofrecer menores pagos de cuotas mensuales en los créditos otorgados.

Sobre este tema y de forma conjunta, los autores de este documento dan seguimiento constante a indicadores como la tasa básica pasiva (TBP-BCCR), el tipo de cambio del colón con respeto al dólar (TC) y la tasa efectiva en dólares (TED), elaborados por el BCCR, como componentes macroeconómicos que inciden sobre las decisiones de inversión y endeudamiento, desde la perspectiva de la situación de los créditos, los ahorros y el tipo de cambio; variables de la política financiera y cambiaria que impactan de forma considerable y directa esta toma de decisiones.

El presente trabajo tiene por objetivo estimar, a partir de una metodología del efecto Fisher internacional, la conveniencia de realizar una inversión financiera en colones o dólares, para el período del I trimestre 2016 al I trimestre del 2019.

La metodología utilizada se basa en la construcción de un modelo para la estimación cualitativa y cuantitativa del efecto Fisher internacional a partir de las tasas de interés de referencia, en moneda nacional y extranjera para el caso de Costa Rica, lo que permite evaluar la pertinencia financiera de invertir en colones o en dólares. 
Para el logro de lo anterior, el artículo se divide en seis secciones, la primera está enfocada en los aspectos introductorios del tema, en la cual se incluye la explicación del cálculo del efecto Fisher internacional y las implicaciones que genera la interacción del tipo de cambio, tasa de interés en moneda nacional y extranjera; la segunda se centra en analizar la tasa básica pasiva y su referencia con los créditos; la tercera corresponde al comportamiento del tipo de cambio del colón con respecto al dólar, en la cuarta se realiza un comparativo del efecto Fisher internacional para distintos plazos de tasas de interés; la quinta sección corresponde al análisis de los resultados obtenidos y, en la última, se exponen las conclusiones.

Ahora, con respecto a la teoría de Fisher, esta toma en consideración tres variables macroeconómicas específicas, a saber: tasa de interés, tipo de cambio y depreciación. Esta teoría establece que en el horizonte de tiempo y a mayor plazo, si se presenta un aumento en la tasa de interés de una moneda, esta presentará su comportamiento en la misma dirección del tipo de cambio (depreciación). Al tratarse de una teoría, esta se fundamenta en un mercado perfecto de libre circulación de capitales y de equilibrio, y deberá existir un efecto de paridad o equilibrio entre las tasas de interés de los países y la manifestación de una posible discrepancia o ventaja en cuanto a la tasa de interés; el mismo mercado se encarga de realizar el ajuste a través de movimientos de capitales.

El efecto Fisher internacional toma en consideración los tipos de cambio a la hora de realizar inversión en el exterior de forma tal que se toma la moneda nacional, como por ejemplo el colón costarricense CRC y se realiza la conversión a la moneda extranjera del país en donde se realizará dicha inversión, como pueden ser los dólares estadounidenses USD. Este capital se invierte en un tiempo " $n$ " definido por lo que se logra obtener un rendimiento promedio. Una vez que llega el vencimiento del capital invertido en el país extranjero (Estados Unidos) se obtiene una ganancia financiera en moneda extranjera más la recuperación del capital invertido. Para darle final al proceso se debe convertir la inversión y réditos nuevamente en moneda local, para que se cumpla con la propuesta internacional de Fisher.

La Figura 1 se construye para sistematizar el efecto Fisher internacional y facilita la comprensión de dicho modelo. 


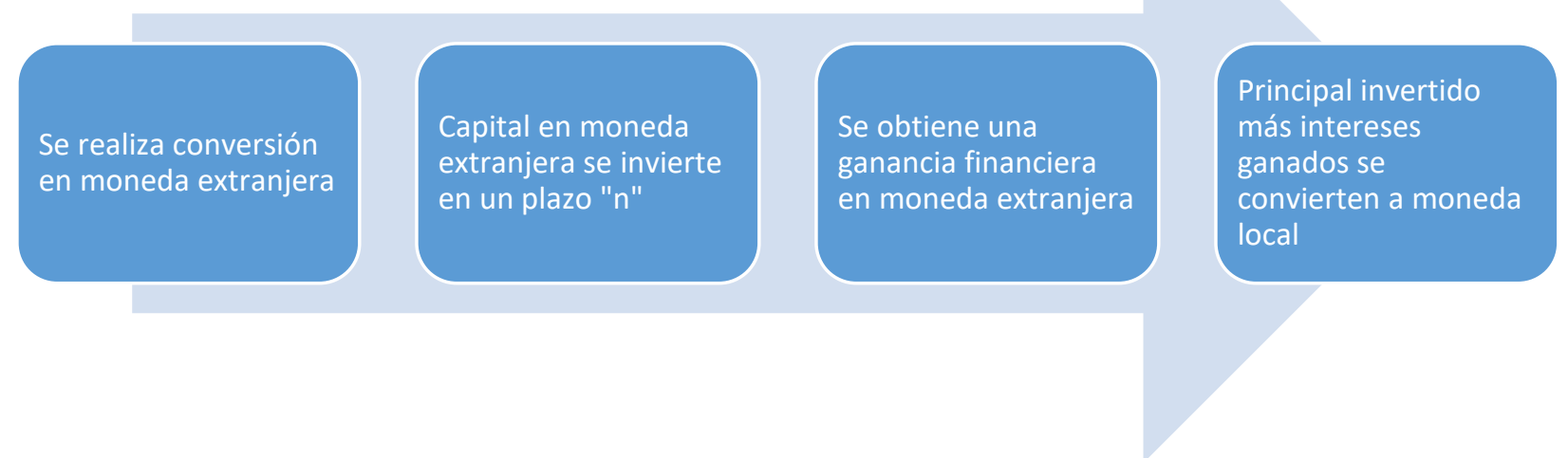

Figura 1. Ciclo de cumplimiento del efecto Fisher internacional. Fuente: elaboración propia.

\section{Tasa básica pasiva y créditos}

Los autores de este artículo han venido dando seguimiento a la información de la tasa básica pasiva del Banco Central de Costa Rica (TBPBCCR) desde el nacimiento de este indicador, en marzo de 1981, por la importancia empírica para la toma de decisiones financieras y económicas a partir del efecto Fisher.

Es importante mencionar que en octubre de 2015 la metodología de cálculo de esta tasa fue modificada por la Junta Directiva del BCCR (sesión 5703-2015); por una parte, incorpora todas las captaciones a plazo en colones, y no solo las de 150 a 210 días como se consideraba antes de la modificación mencionada; y por otra, trabaja con una población de referencia excluyendo tasas de interés atípicas, con el objetivo de que esas tasas en las operaciones de captación no distorsione este cálculo, procura, así, evitar los efectos adversos sobre la eficiencia del sistema financiero.

En cuanto al comportamiento de la tasa básica pasiva (TBP) se tiene que el punto más alto se presentó en febrero de 1991, con un nivel del 35 \%, el cual responde a un proceso de mayor demanda de fondos prestables, producto del crecimiento económico que mostraba el país; mientras el más bajo fue en abril y mayo de 2008 , con un nivel del $4.25 \%$, producto de los efectos primarios de lo que se denominaría la crisis económica mundial.

Esta dinámica de fondos prestables se puede comprender mejor observando la tasa de variación anual en el producto interno bruto nominal, como se muestra en la Figura 2. 


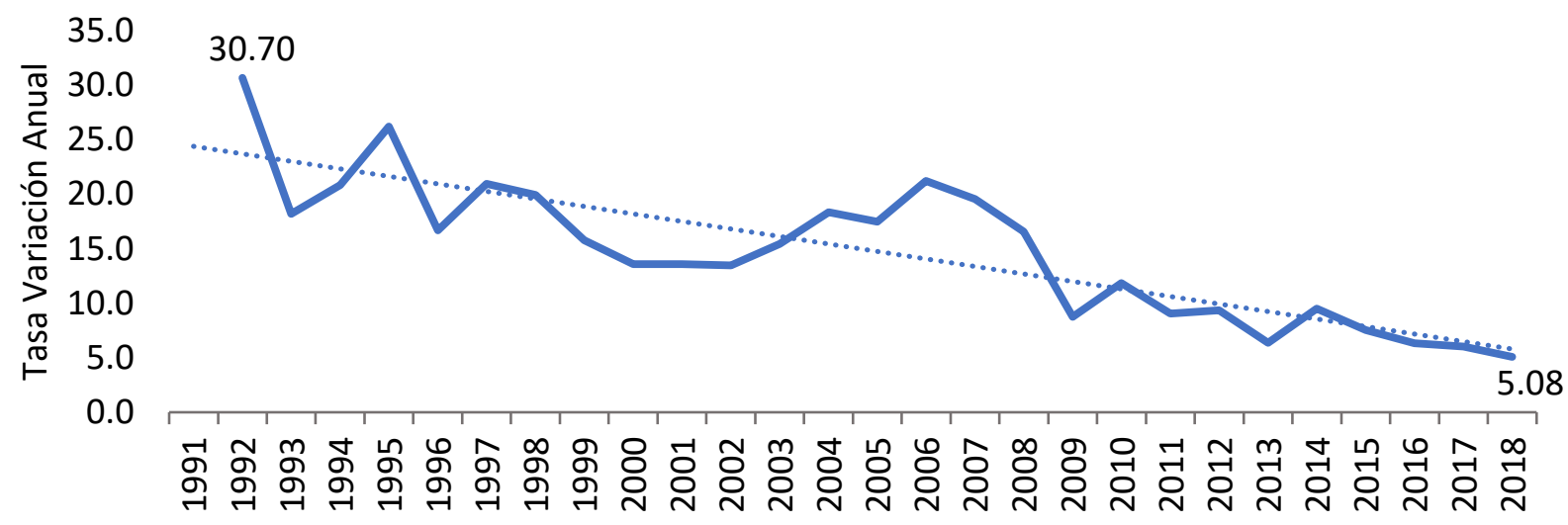

Años

Figura 2. Variación interanual del producto interno bruto nominal a precios de mercado. Período 1991-2018.Fuente: elaboración propia a partir de datos del Banco Central de Costa Rica

Además, el uso de la TBP calculada por el BCCR es trascendental para las entidades financieras, por ser un indicador económico utilizado para referenciar las tasas de interés activas (préstamos) y las pasivas (certificados).

Ejemplo: si la TBP se ubica en un $5 \%$ y la entidad financiera decide referenciar el préstamo o inversión a partir de TBP+3 p.p., lo que se obtendría es una tasa de $8 \%$ para cada cliente potencial, programándose fluctuaciones de acuerdo con el comportamiento de esta tasa, con la consecuente afectación a préstamos e inversiones.

Por ello, en el sistema financiero nacional existe una gran gama de créditos dirigidos a diferentes necesidades y segmentos de mercado de la población, que vienen a satisfacer sus insuficiencias de liquidez. Es importante resaltar que la finalidad del negocio de los intermediarios financieros es el de realizar captaciones a una tasa de interés y colocar esos dineros a una tasa de interés mayor, lo que genera el llamado margen de intermediación que les permite a las instituciones financieras no solo cubrir sus costos operativos, sino también obtener ganancias de capital.

En lo que a créditos se refiere, el sistema financiero nacional ofrece diversos productos dirigidos a suplir diversas necesidades de liquidez que se categorizan en dos grupos:

- Créditos de inversión: Catalogados como una inversión y no como gasto o consumo, en esta categoría se incluyen todos aquellos créditos dirigidos a mejorar el nivel de vida de los individuos. Esta categoría de crédito se caracteriza por poseer las tasas de interés más bajas y es, precisamente, a las que los intermediarios financieros les aplican mecanismos de pagos más flexibles, a fin de facilitar y hacer sostenible la vida financiera de las operaciones. 
Acá se incluyen aquellos créditos dirigidos como lo son vivienda, gastos médicos, pago de estudios, compra de terrenos y, más recientemente, compra de vehículo, ya que, a diferencia de años atrás, la necesidad de transporte más que un lujo hoy se cataloga como una necesidad básica de la población y un elemento central para mejorar la competitividad del país, pues según el informe de la Contraloría General de la República, denominado Resultado de servicios de transporte y carreteras de febrero de 2018, el rango promedio per cápita de uso del transporte público se estima esta entre una hora y cinco minutos y dos horas diarias, lo que también genera una pérdida de eficiencia en el capital humano.

- Créditos para consumo: En contraposición con el punto anterior, existe una modalidad de créditos que son conducentes al gasto o consumo; créditos que, por su naturaleza, condiciones y tipos de garantía, necesariamente los entes intermediarios financieros les aplican una tasa de interés totalmente diferenciada y relativamente elevada con respecto a otros tipos de créditos.

Dentro de esta categoría se cuenta con todos aquellos créditos orientados a cancelar o refundir créditos, viajes, compra de electrodomésticos, entre otros.

La relevancia de estos créditos, vivienda y consumo se puede apreciar en la Tabla 2, donde al calcular el peso relativo de estos rubros para diciembre 2017 y diciembre 2018 en el crédito total al sector privado no financiero se puede comprobar que mantienen un peso relativo del $28 \%$ y el $33 \%$, respectivamente en ambos años; concentrando ambos tipos de créditos el $50 \%$ de la cartera total.

Adicionalmente, se vive en el país un fenómeno a partir del endeudamiento a través de tarjetas de crédito, como se evidencia en los estudios trimestrales del Ministerio de Economía, Industria y Comercio (MEIC), donde, si bien estas tarjetas se han convertido en un mecanismo de pago en el comercio de bienes y servicios, según el último estudio a enero 2019, se cuenta con treinta emisores de tarjetas en el país y una deuda total de 1.450.344 millones de colones a este mes, lo que equivale a un 0,04 \% del producto interno bruto (PIB) correspondiente al año 2018.

Además, a partir del informe para enero 2019 (DAEM-INF-002-19) se tienen 461 tipos de tarjetas de crédito, lo que significaba 2.933.787 tarjetas en circulación o, dicho en otras palabras, tarjetas en los bolsillos de la ciudadanía.

La anterior situación ha generado un vector de precios, los cuales se pueden observar en la Figura 3 , donde se puede comprobar que el rango de $40 \%$ al 49,9\% representa la mayor participación relativa, de un 69,1\% del total 262 plásticos que circulaban en enero de 2019. Mientras que los otros rangos muestran participaciones relativas menores, del 21,4 \% para el rango de $30 \%$ a $39.9 \%$ y de $9,5 \%$ para el rango de tasas de interés anuales entre el $19 \%$ y $29,9 \%$.

Carlos Leonardo Arguedas Campos y Gustavo Vallejo Esquivel

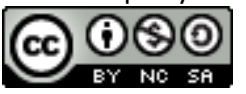

Revista Economía y Sociedad by Universidad Nacional is licensed under a CreativeCommons Reconocimiento-NoComercial- 
Tabla 2

Crédito otorgado por el sistema financiero al sector privado no financiero por actividad económica. Saldo a fin de mes en millones de colones y peso relativo

\begin{tabular}{lrrrr}
\hline \multicolumn{1}{c}{ Año / } & \multicolumn{2}{c}{ Diciembre 2017 } & \multicolumn{2}{c}{ Diciembre 2018 } \\
\hline \multicolumn{1}{c}{ Sector } & \multicolumn{1}{c}{ Monto } & Peso relativo & \multicolumn{1}{c}{ Monto } & Peso relativo \\
\hline Total & $\mathbf{1 9 . 9 5 6 . 1 4 2 , 4 2}$ & $\mathbf{1 0 0 \%}$ & $\mathbf{2 1 . 1 8 2 . 7 7 0 , 2 9}$ & $\mathbf{1 0 0 \%}$ \\
Agricultura & $428.371,80$ & $2 \%$ & $446.655,52$ & $2 \%$ \\
Ganadería & $208.695,60$ & $1 \%$ & $211.960,41$ & $1 \%$ \\
Pesca & $5.532,80$ & $0 \%$ & $5.281,78$ & $0 \%$ \\
Industria & $752.559,50$ & $4 \%$ & $784.024,20$ & $4 \%$ \\
Vivienda & $\mathbf{5 . 5 1 8 . 7 2 3 , 2 0}$ & $\mathbf{2 8 \%}$ & $\mathbf{5 . 8 6 4 . 5 0 4 , 9 4}$ & $\mathbf{2 8 \%}$ \\
Construcción & $483.751,40$ & $2 \%$ & $531.116,54$ & $3 \%$ \\
Turismo & $382.198,70$ & $2 \%$ & $409.463,87$ & $2 \%$ \\
Comercio & $2.219 .862,00$ & $11 \%$ & $2.369 .270,62$ & $11 \%$ \\
Servicios & $2.574 .181,90$ & $13 \%$ & $2.771 .861,35$ & $13 \%$ \\
Consumo & $\mathbf{6 . 5 2 2 . 3 6 9 , 1 0}$ & $\mathbf{3 3 \%}$ & $\mathbf{6 . 9 6 2 . 2 3 9 , 7 5}$ & $\mathbf{3 3 \%}$ \\
Otras actividades: & $859.896,40$ & $4 \%$ & $826.391,30$ & $4 \%$ \\
$\quad$ Electricidad & $478.349,01$ & & $415.102,90$ & \\
Transportes & $38.902,34$ & & $237.372,52$ & \\
Depósitos y almac. & $8.190,02$ & & $2.066,01$ & $171.849,88$ \\
Otras & $134.455,07$ & & & \\
\hline
\end{tabular}

Fuente: Elaboración propia a partir de datos del BCCR.

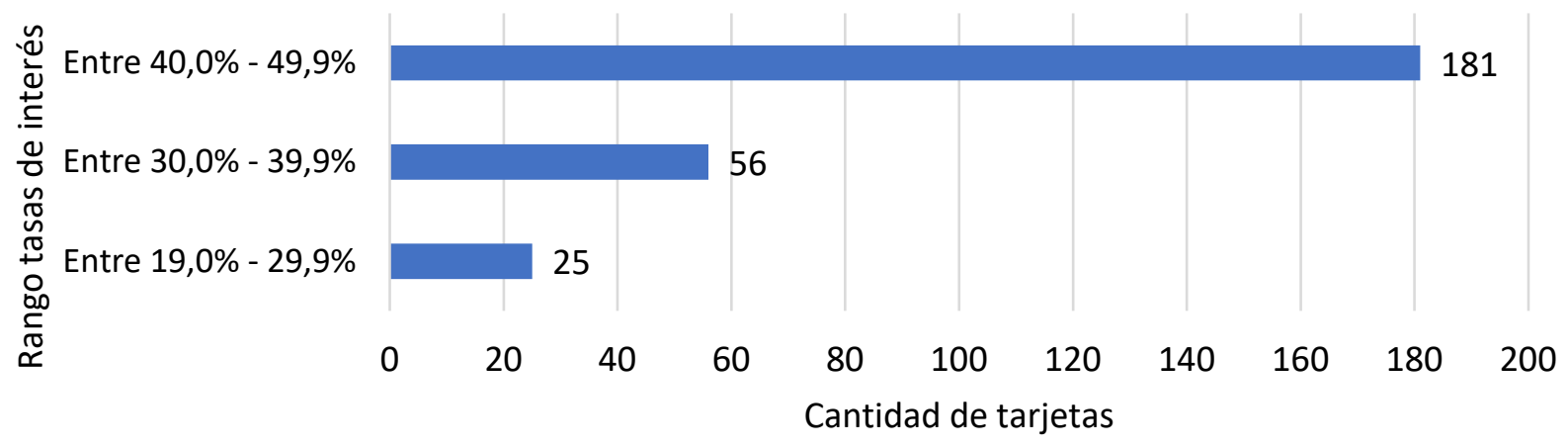

Figura 3. Relación de cantidad de tarjetas en circulación y rangos de tasas de interés anual en colones, al 31 de enero 2019. Fuente: elaboración propia a partir del Estudio trimestral de tarjetas de crédito del 2019 del Ministerio de Economía, Industria y Comercio de Costa Rica.

Producto de estos rangos de tasas de interés y del uso que se les da a las tarjetas de crédito, se ha generado un incremento en la morosidad, específicamente, la mayor a 90 días, entre los años 2018 al 2019 como se muestra en la Figura 4 correspondiente a la evolución de la morosidad durante el período 2016 - 2019. 


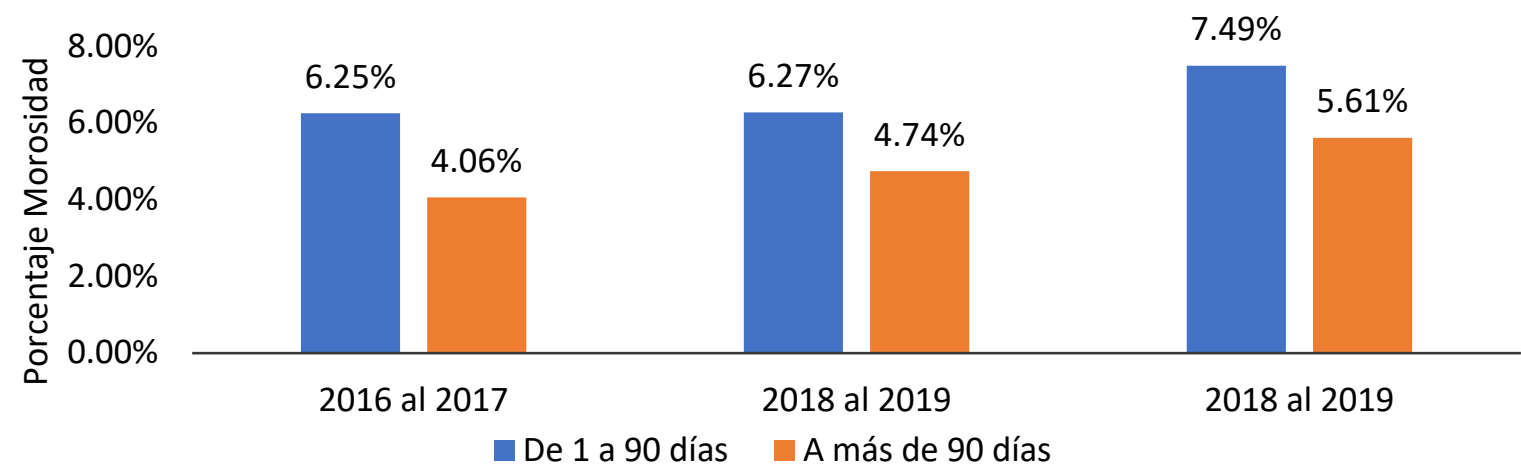

Figura 4. Cartera en morosidad de tarjetas de crédito. Variación interanual a enero de cada año. 2016 - 2019. Fuente: elaboración propia a partir de datos del sitio web del Ministerio de Economía, Industria y Comercio de Costa Rica.

Volviendo a la exposición sobre los indicadores relevantes de este artículo, se tiene la tasa básica pasiva en la Figura 5 donde se presenta el comportamiento histórico de esta tasa para el horizonte de tiempo comprendido del 26 de marzo de 1981 al 31 de marzo de 2019. Esta figura muestra la situación económica por la que pasó el país a inicios de los años ochenta en la administración Carazo Odio; la crisis que se presentó con las financieras el último quinquenio de los años ochenta, el cierre del Banco Anglo Costarricense en 1994, la crisis del sector cooperativo de finales de los años noventa, la crisis inmobiliaria del 2008, el proceso de cierre de Bancrédito en mayo 2017, la situación con el crédito otorgado por el Banco de Costa Rica para la importación de cemento chino en el año 2016, entre los factores que se consideran, incidieron directamente en el comportamiento de la tasa básica pasiva calculada por el BCCR.

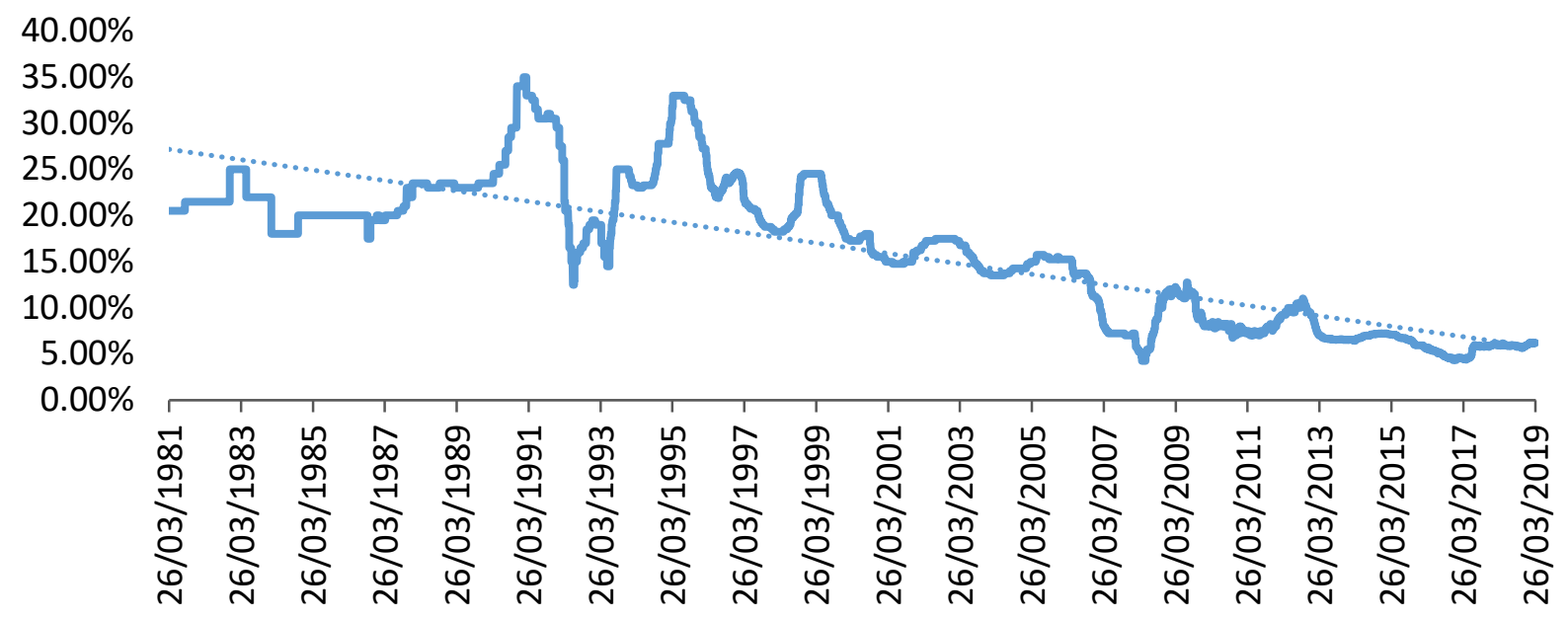

Figura 5. Tasa básica pasiva. Del 26 de marzo de 1981 al 31 de marzo de 2019. Fuente: elaboración propia con datos del BCCR. 


\section{Tipo de cambio del dólar con respecto al colón}

El tipo de cambio del dólar estadounidense con respecto al colón costarricense históricamente ha mantenido una tendencia creciente con ciertos picos de comportamiento altos como el presentado en el año 2017, cuando el tipo de cambio, según datos obtenidos del BCCR, subió de forma apresurada de un precio de venta promedio de los primeros veinticuatro días del mes de mayo de $\$ 578.28$ a $\$ 598.48 / \$$ el 25 de mayo, el nivel más alto hasta esa fecha; también se ubicó ese mismo tipo de cambio al cierre de julio de 2017 en un precio de venta de $\$ 575.17 / \$$ y al 28 agosto en $\$ 577.27 / \$$, situación generada principalmente por un proceso de especulación cambiaria y de expectativas económicas producto de la discusión fiscal del país.

Durante el año 2018 y hasta el primer semestre 2019, el tipo de cambio ha mantenido un comportamiento que, a criterio de los autores, ha sido estable al mantenerse cercano al precio de $\$ 570$ y aunque ha presentado ciertos picos, estos no han sido tan alarmantes como los presentados en mayo de 2017.

Con referencia al tipo de cambio, se acota que, a partir del 30 de enero de 2015, la Junta Directiva del BCCR, mediante artículo 5 de la sesión 5677-2015, tomó el acuerdo de migrar la metodología del tipo de cambio, pasando de un régimen de banda cambiaria a flotación administrada en donde este último es determinado primordialmente por la oferta y demanda de la divisa extranjera.

Ahora, con el objetivo de poder introducir el cálculo del efecto Fisher internacional", en la siguiente sección, se muestra en la Figura 6 el comportamiento del tipo de cambio de venta del colón con respecto al dólar estadounidense.

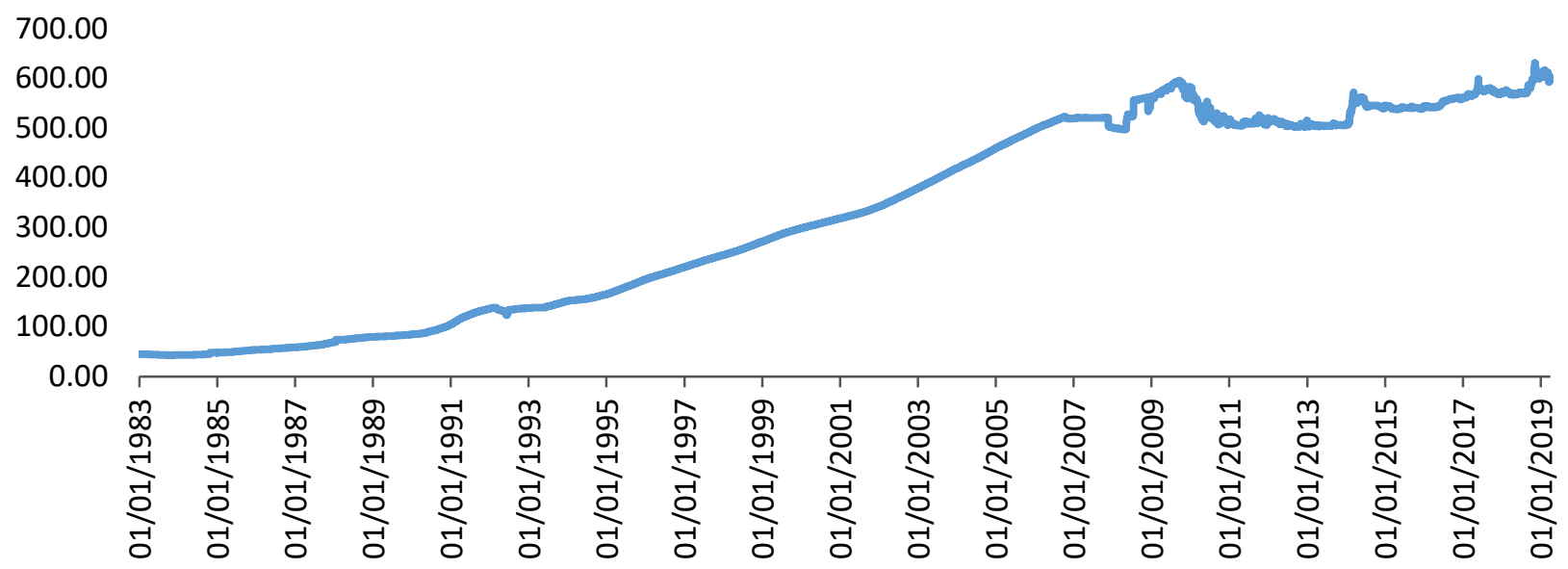

Figura 6. Tipo de cambio de venta, colones por dólar estadounidense. Del 26 de marzo de 1981 al 31 de marzo de 2019. Fuente: Elaboración propia con datos del BCCR. 


\section{Efecto Fisher internacional (EFI)}

Tal y como se explicó en el primer apartado, la teoría de Fisher toma en consideración tres variables macroeconómicas: tasa de interés, tipo de cambio y depreciación; por ello, para explicar el efecto Fisher internacional (EFI), se toma como base el primer trimestre del año 2015, luego se realizan proyecciones del tipo de cambio de forma trimestral, durante el período comprendido entre el I trimestre 2016 y I trimestre 2019.

A partir de la información disponible para Costa Rica, los autores de este documento han creado un modelo para hacer interactuar las variables económicas y medir el efecto que tiene el tipo de cambio y las tasas de interés de las inversiones en la paridad del tipo de cambio a futuro "forward" o efecto Fisher internacional (EFI). Las variables utilizadas son las siguientes: Tasas de interés netas del sector público costarricense, tasas de interés del Tesoro de los Estados Unidos de América, tasas negociadas en la Bolsa Nacional de Valores, tipo de cambio venta publicado por el BCCR y cálculo de la depreciación con base en diciembre del último período.

La fórmula que se presenta a continuación (efecto Fisher internacional, EFI) es la que se utiliza para calcular el tipo de cambio a futuro (Forward) a una fecha determinada a partir de un tipo de cambio inmediato (Spot), que para este caso es un tipo de cambio que consigue un equilibrio perfecto y logra paridad de tasas de interés.

TCf $=$ TCs $*\left(\frac{1+\text { Interés local }}{1+\text { Interés externo }}\right)$

\section{En donde:}

TC $\boldsymbol{f}=$ Tipo de cambio "Forward" proyectado al trimestre en estudio.

TCs= Tipo de cambio "Spot" correspondiente al trimestre anterior al de estudio.

Interés local= Tasa de interés local aplicada a colones

Interés externo= El equivalente de la tasa efectiva en dólares expresada en su equivalente en colones de acuerdo con la siguiente fórmula:

$$
\mathbf{i} \Phi=((\mathbf{1}+\mathbf{i} \$) *(\mathbf{1}+\text { depreciación }))-\mathbf{1}) * \mathbf{1 0 0})
$$

\section{En donde:}

ic= Equivalente en tasa de interés en colones

i\$= Tasa efectiva en dólares

Depreciación= Variación porcentual del tipo de cambio del colón con respecto al dólar, según el último trimestre en estudio. 
A continuación, se presenta el cálculo de la proyección del tipo de cambio a futuro "forward" tomando como ejemplo el primer trimestre del año 2016 en donde se tienen los siguientes datos:

\section{Variables:}

- Tipo de cambio al $1 / 1 / 2016=544.87 / \$$

- Tasa de interés en colones $=4.048 \%$

- Tasa de interés en dólares $=1.050 \%$

- Depreciación $=-0.121 \%$

- Tipo de cambio al 31/03/2016= $\$ 542.23 / \$$

- Equivalente en colones de la tasa en dólares de $0.748 \%=0.260 \%$

\section{Paso I (Equivalencia de tasa de interés de dólares a tasa en colones)}

$$
\begin{aligned}
\boldsymbol{i} \Phi & =((1+i \$) *(1+\text { depreciación }))-1) * 100) \\
\boldsymbol{i} \Phi & =((1+1.050 \%) *(1+(-0.121 \%))-1) * 100) \\
\boldsymbol{i} \Phi & =0.928 \%
\end{aligned}
$$

\section{Paso II (Cálculo del efecto Fisher internacional, EFI)}

$$
T C f=T C s *\left(\frac{1+\text { Interés local }}{1+\text { Interés externo }}\right)
$$

$\boldsymbol{T C f}=544.87 *\left(\frac{1+4.048 \%}{1+0.928 \%}\right)$

$\boldsymbol{T C f}=561.72$

Se parte de un tipo de cambio "spot" (inmediato) al primero de enero 2016 de $\$ 544.87$ y se obtiene un tipo de cambio "forward" de $\$ 561.72 / \$$, cifra que se relaciona con el tipo de cambio de venta publicado para el 31 de marzo de 2016 de $\$ 542.23$. Para este caso, el resultado obtenido corresponde a una diferencia de $\$ 19.49$ por debajo de la proyección, de acuerdo con el efecto Fisher internacional.

Con los números obtenidos del modelo, se establece que para el primer semestre de 2016; en esa coyuntura, las que lograron un mayor incentivo por sus inversiones fueron las inversiones en colones, ya que además de ganar una tasa de interés similar a la de dólares, obtenían a esa fecha un tipo de cambio por debajo del paridad de tasa de interés, lo que eventualmente provocó una ganancia de capital en comparación con las inversiones en dólares; lo anterior significa que quienes invirtieron en colones en el primer trimestre de 2016 al cabo de tres meses obtenían un 
tipo de cambio por debajo del de paridad de tasa de interés, lo que les permitía comprar más dólares con los mismos colones.

A continuación, se presentan cinco resultados del modelo expuesto, a fin de determinar si con las variables de tipo de cambio "spot", tasas de interés y depreciación, se cumple con la paridad del efecto Fisher internacional, mencionado en el párrafo anterior.

\section{A. Cálculo del efecto Fisher internacional (EFI) con tasas de interés netas a dos años}

Para obtener el cálculo del EFI se requiere de la utilización de las tasas de interés domésticas y extranjeras, para lo cual se toman las del sector público costarricense y su homóloga en dólares correspondientes a las tasas del Tesoro de los Estados Unidos de América, ambas publicadas por el BCCR, así como las variaciones en el tipo de cambio del período en estudio, como se muestra en la Tabla 3.

Tabla 3

Tasas de interés netas a dos años. Del IV trimestre 2015 al I trimestre 2016. Por trimestre

\begin{tabular}{lccc}
\hline \multicolumn{1}{c}{ Fecha de corte } & $\begin{array}{c}\text { Porcentaje de depreciación } \\
\text { trimestral }\end{array}$ & $\begin{array}{c}\text { Tasas de interés netas Sector } \\
\text { público CRC }\end{array}$ & $\begin{array}{c}\text { Tasas del Tesoro } \\
\text { de EUA }\end{array}$ \\
\hline IV Trimestre 2015 & $0,000 \%$ & $4,048 \%$ & $1,050 \%$ \\
I Trimestre 2016 & $0,000 \%$ & $3,634 \%$ & $0,748 \%$ \\
II Trimestre 2016 & $0,000 \%$ & $3,472 \%$ & $0,584 \%$ \\
III Trimestre 2016 & $0,000 \%$ & $3,719 \%$ & $0,764 \%$ \\
IV Trimestre 2016 & $0,000 \%$ & $3,709 \%$ & $1,214 \%$ \\
I Trimestre 2017 & $0,000 \%$ & $3,879 \%$ & $1,256 \%$ \\
II Trimestre 2017 & $0,000 \%$ & $1,384 \%$ \\
III Trimestre 2017 & $0,000 \%$ & $6,241 \%$ & $1,486 \%$ \\
IV Trimestre 2017 & $0,000 \%$ & $6,365 \%$ & $1,885 \%$ \\
I Trimestre 2018 & $0,000 \%$ & $6,280 \%$ & $2,285 \%$ \\
II Trimestre 2018 & $0,000 \%$ & $6,419 \%$ & $2,530 \%$ \\
III Trimestre 2018 & $0,000 \%$ & $6,686 \%$ & $2,821 \%$ \\
IV Trimestre 2018 & $0,000 \%$ & $2,518 \%$ \\
I Trimestre 2019 & $-0,346 \%$ & $6,573 \%$ & $2,340 \%$ \\
\hline
\end{tabular}

Nota: elaboración propia con datos del BCCR.

Dentro del análisis de la información para el presente artículo, a continuación, se presenta la Tabla 4, la que toma en consideración los tipos de cambio publicados por el BCCR para transacciones a plazo de dos años tanto en colones como en dólares, modelo que se obtiene de los datos alcanzados de la última columna, la diferencia entre y el proyectado según el EFI. Si el dato obtenido es negativo, existe un incentivo para invertir en dólares, puesto que, de acuerdo con las variables, el tipo de cambio del colón estaría por debajo el tipo de cambio a futuro, lo que genera una ganancia adicional al ente inversionista en dólares, vía tipo de cambio, ya que eventualmente recibiría, aparte de los intereses ganados por la inversión, más colones de los que debería recibir 
en una condición de equilibrio de tasas de interés o EFI, como se expuso en párrafos anteriores, con el ejemplo de la ecuación del efecto Fisher internacional.

Tabla 4

Cálculo del EFI, aplicando tasas de interés a dos años plazo. Del IV trimestre 2015 al I trimestre 2019

\begin{tabular}{lccc}
\hline Fecha de Corte & $\begin{array}{c}\text { Tipo de cambio } \\
\text { venta }\end{array}$ & $\begin{array}{c}\text { Proyección efecto Fisher } \\
\text { internacional }\end{array}$ & $\begin{array}{c}\text { Diferencia tipo de cambio venta y } \\
\text { proyectado }\end{array}$ \\
\hline IV Trimestre 2015 & 544,87 & Período Base & Período Base \\
I Trimestre 2016 & 542,23 & 561,72 & $-19,49$ \\
II Trimestre 2016 & 554,20 & 560,48 & $-6,28$ \\
III Trimestre 2016 & 558,80 & 560,51 & $-1,71$ \\
IV Trimestre 2016 & 561,10 & 560,85 & 0,25 \\
I Trimestre 2017 & 567,34 & 558,30 & 9,04 \\
II Trimestre 2017 & 579,87 & 575,64 & 4,23 \\
III Trimestre 2017 & 574,13 & 587,98 & $-13,85$ \\
IV Trimestre 2017 & 572,56 & 588,08 & $-15,52$ \\
I Trimestre 2018 & 569,31 & 585,30 & $-15,99$ \\
II Trimestre 2018 & 570,08 & 595,70 & $-25,62$ \\
III Trimestre 2018 & 585,80 & 595,77 & $-9,97$ \\
IV Trimestre 2018 & 611,75 & 593,45 & 18,30 \\
I Trimestre 2019 & 602,36 & 593,98 & 8,38 \\
\hline
\end{tabular}

Fuente: elaboración propia con datos del BCCR.

En la Figura 7 se muestra el comportamiento del tipo de cambio de venta y sus proyecciones de acuerdo con las condiciones de mercado presentadas en el momento para tasas de interés a dos años, lo que permite calcular el tipo de cambio futuro según tasas de interés y depreciación proyectada, todo de forma trimestral a partir del primer trimestre del año 2016.

Nótese que la línea celeste, en la Figura 7, corresponde al tipo de cambio venta, cada vez que se ubica por encima de la línea anaranjada, que corresponde a la proyección del tipo de cambio según el EFI. Se puede afirmar que aquellos sujetos que han invertido en dólares y no en colones han recibido un incentivo mayor por sus inversiones, ya que aparte de que obtienen una tasa de interés en el eventual caso de que las conviertan a colones, ganarán un ingreso adicional, pues no se presentaría una condición de paridad entre las tasas de interés relacionada con la depreciación.

Las mayores disparidades entre tasas de interés y tipos de cambio se presentaron en el I trimestre 2017, IV trimestre 2018 y I trimestre 2019 con precios por encima del tipo obtenido por el modelo de $\$ 9.04, \$ 18.30$ y $\ 8.38$, respectivamente, lo que favoreció a inversionistas en dólares. 
Por otro lado, quienes invirtieron en colones durante el I trimestre 2016, I trimestre 2018 y II trimestre 2018, lograron precios de \$19.49, \$15.99 y \$25.62 por debajo del tipo de cambio obtenido con el modelo, con la eventualidad de que podían comprar dólares por esos montos por debajo del tipo de cambio a futuro.

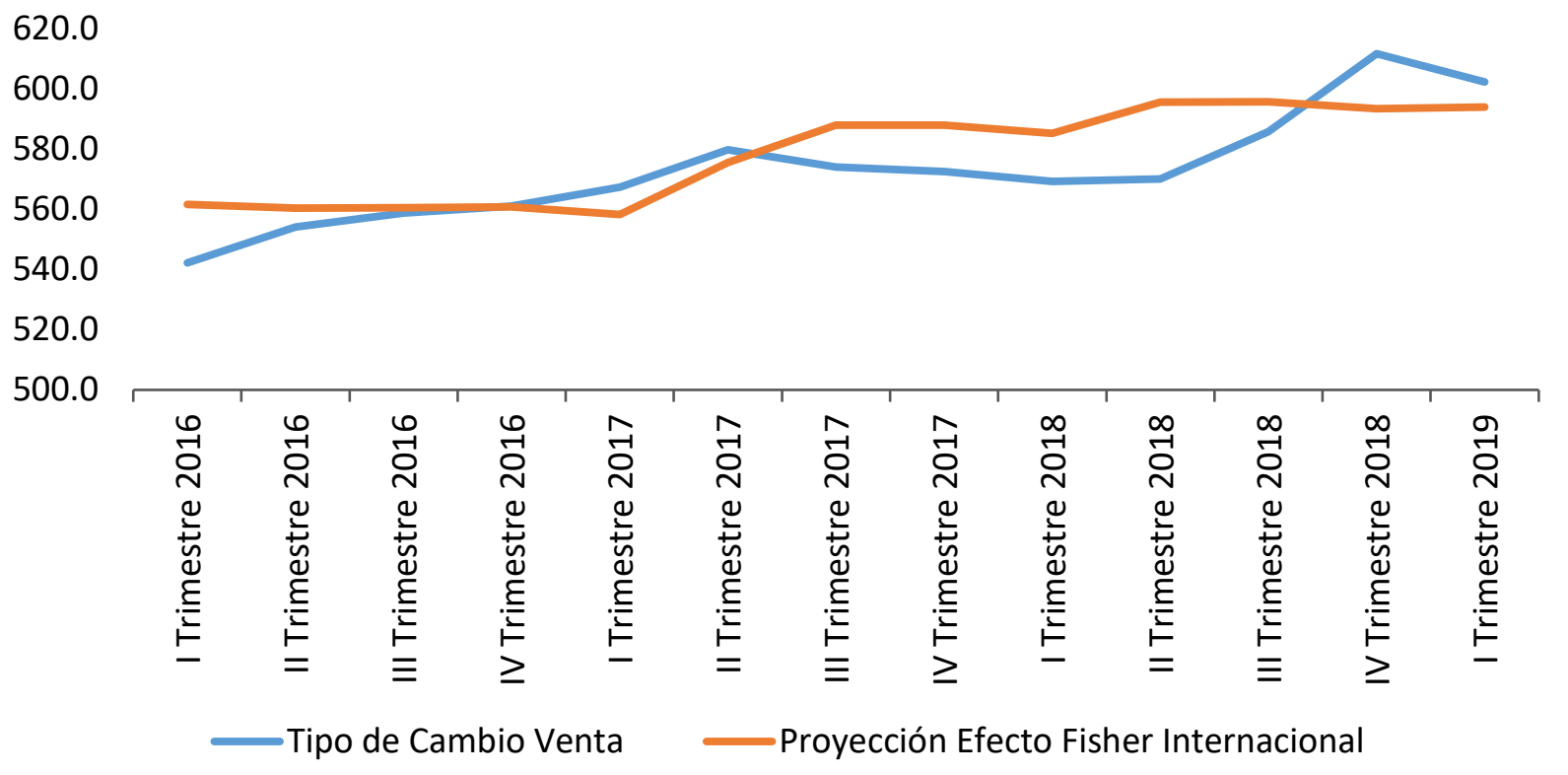

Figura 7. Proyección tipo de cambio y efecto Fisher internacional EFI. Tasas de interés a dos años. Del I trimestre 2016 al I trimestre 2019. Fuente: elaboración propia con datos del BCCR.

Con la aplicación del modelo, con respecto a las tasas de interés netas del sector público costarricense interrelacionadas con las tasas del Tesoro de los Estados Unidos de América, se obtuvo un promedio de variación de la diferencia del tipo de cambio de acuerdo con el efecto Fisher internacional, para el período comprendido del primer trimestre de 2016 al primer trimestre de 2019 de $\mathbf{c}-5.25$, donde se observan datos con nivel máximo de variación correspondiente a $\$ 18.30$ en el IV trimestre de 2018 y un nivel mínimo de $\$-25.62$ en el II trimestre 2018.

\section{B. Cálculo del EFI con tasas de interés netas a cinco años}

Siguiendo la misma metodología utilizada en el apartado anteriormente, se procede a calcular el efecto Fisher internacional, aplicando tasas de interés netas para un plazo de cinco años.

En la Tabla 5 se presenta la información de las tasas de interés netas respectivas para un plazo de cinco años. 
Tabla 5

Tasas de interés netas a cinco años. Del VI trimestre 2015 al I trimestre 2016. Por trimestre.

\begin{tabular}{lccc}
\hline \multicolumn{1}{c}{ Fecha de corte } & $\begin{array}{c}\text { Porcentaje de } \\
\text { depreciación trimestral }\end{array}$ & $\begin{array}{c}\text { Tasas de interés netas } \\
\text { sector público CRC }\end{array}$ & $\begin{array}{c}\text { Tasas del Tesoro } \\
\text { de E.U.A. }\end{array}$ \\
\hline IV Trimestre 2015 & $-0,121 \%$ & $5,398 \%$ & $1,761 \%$ \\
I Trimestre 2016 & $-0,485 \%$ & $5,485 \%$ & $1,205 \%$ \\
II Trimestre 2016 & $1,712 \%$ & $5,245 \%$ & $1,000 \%$ \\
III Trimestre 2016 & $2,557 \%$ & $5,340 \%$ & $1,150 \%$ \\
IV Trimestre 2016 & $2,979 \%$ & $5,126 \%$ & $1,955 \%$ \\
I Trimestre 2017 & $1,112 \%$ & $5,310 \%$ & $1,922 \%$ \\
II Trimestre 2017 & $3,345 \%$ & $6,702 \%$ & $1,889 \%$ \\
III Trimestre 2017 & $2,322 \%$ & $7,215 \%$ & $1,937 \%$ \\
IV Trimestre 2017 & $2,042 \%$ & $7,276 \%$ & $2,070 \%$ \\
I Trimestre 2018 & $-0,568 \%$ & $7,348 \%$ & $2,596 \%$ \\
II Trimestre 2018 & $-0,433 \%$ & $7,714 \%$ & $2,739 \%$ \\
III Trimestre 2018 & $2,312 \%$ & $7,818 \%$ & $2,954 \%$ \\
IV Trimestre 2018 & $6,845 \%$ & $8,181 \%$ & $2,512 \%$ \\
I Trimestre 2019 & $-1,535 \%$ & $8,400 \%$ & $2,258 \%$ \\
\hline
\end{tabular}

Fuente: elaboración propia con datos del BCCR.

Seguidamente se procede a proyectar el tipo de cambio para un escenario de cinco años, para las tasas de interés del sector público costarricense y las tasas del Tesoro de los Estados Unidos de América, según se muestra en la Tabla 6.

Tabla 6

Cálculo del Fisher internacional EFI. Aplicando tasas de interés a cinco años plazo. Del IV trimestre 2015 al I trimestre 2019

\begin{tabular}{|c|c|c|c|}
\hline Fecha de corte & Tipo de cambio venta & $\begin{array}{c}\text { Proyección efecto } \\
\text { Fisher internacional }\end{array}$ & $\begin{array}{c}\text { Diferencia tipo de cambio } \\
\text { venta y proyectado }\end{array}$ \\
\hline IV Trimestre 2015 & 544,87 & Período base & Período base \\
\hline I Trimestre 2016 & 542,23 & 565,03 & $-22,80$ \\
\hline II Trimestre 2016 & 554,20 & 567,91 & $-13,71$ \\
\hline III Trimestre 2016 & 558,80 & 567,77 & $-8,97$ \\
\hline IV Trimestre 2016 & 561,10 & 567,44 & $-6,34$ \\
\hline I Trimestre 2017 & 567,34 & 561,82 & 5,52 \\
\hline II Trimestre 2017 & 579,87 & 579,75 & 0,12 \\
\hline III Trimestre 2017 & 574,13 & 587,61 & $-13,48$ \\
\hline IV Trimestre 2017 & 572,56 & 590,15 & $-17,59$ \\
\hline I Trimestre 2018 & 569,31 & 589,72 & $-20,41$ \\
\hline II Trimestre 2018 & 570,08 & 599,08 & $-29,00$ \\
\hline III Trimestre 2018 & 585,80 & 600,29 & $-14,49$ \\
\hline IV Trimestre 2018 & 611,75 & 599,61 & 12,14 \\
\hline I Trimestre 2019 & 602,36 & 604,22 & $-1,86$ \\
\hline
\end{tabular}

Fuente: elaboración propia con datos del BCCR. 
A partir de los resultados del modelo de la Tabla 6, se obtiene que cada vez que la diferencia entre el tipo de cambio de venta y el proyectado es positiva, el ente inversionista en dólares obtendrá una ganancia producto no solo de los intereses recibidos por la inversión, sino también producto de la conversión de esos dólares a colones, puesto que el tipo de cambio futuro sería mayor al tipo de cambio inmediato; situación contraria sucede, si la diferencia es negativa.

De igual manera que se expuso en el modelo que incluyó las tasas de interés para un plazo de dos años, a continuación, se presenta la Figura 8 que muestra el comportamiento del tipo de cambio de venta y sus proyecciones de acuerdo con las condiciones de mercado presentadas en el momento para tasas de interés a cinco años, lo que permite calcular el tipo de cambio esperado según tasas de interés y depreciación proyectada.

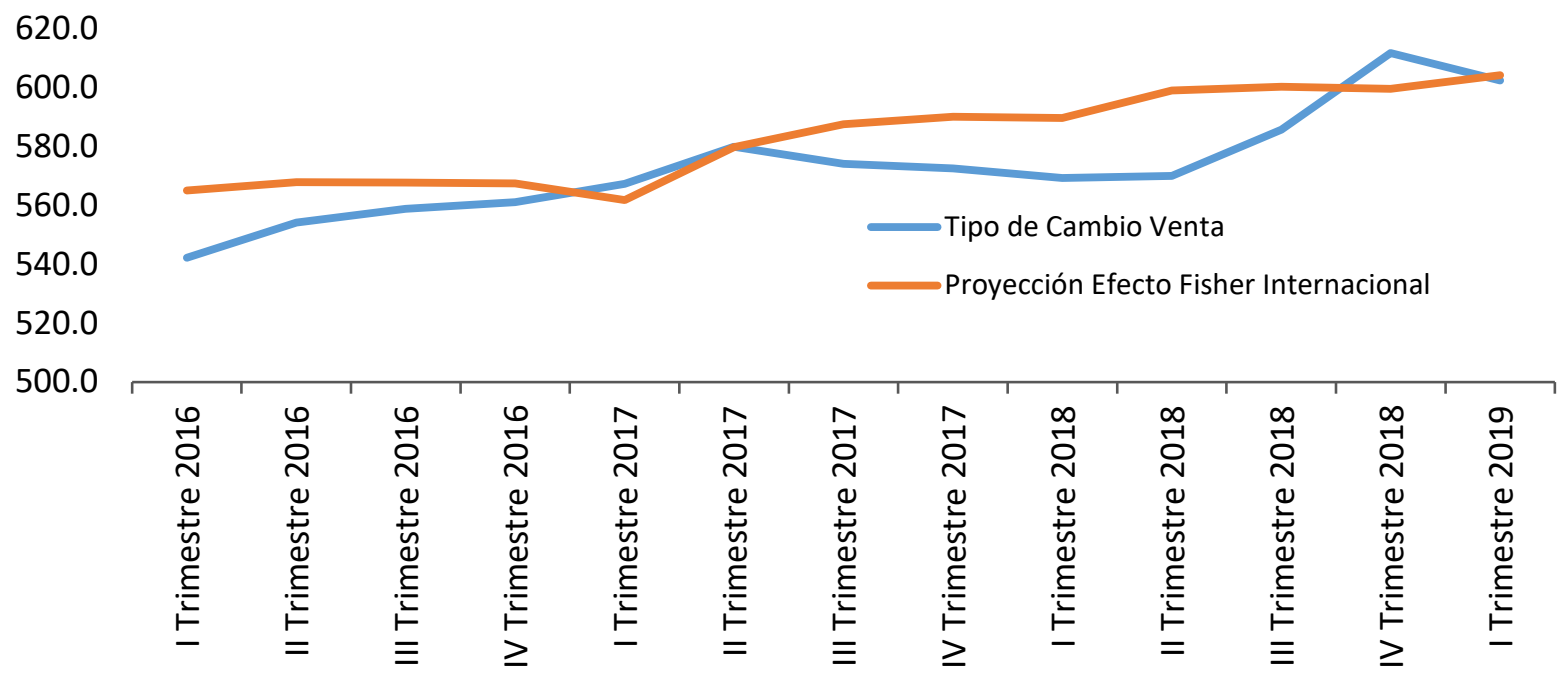

Figura 8. Proyección tipo de cambio y efecto Fisher internacional EFI. Tasas de interés a cinco años del I trimestre 2016 al I trimestre 2019. Fuente: elaboración propia con datos del BCCR.

Al igual que el modelo presentado con anterioridad, si la línea celeste, que corresponde al tipo de cambio de venta, se ubica por encima de la línea anaranjada, que corresponde a la proyección del tipo de cambio según el efecto Fisher internacional, aquellos sujetos que han invertido en dólares han recibido un incentivo mayor por sus inversiones, ya que aparte de que obtienen una tasa de interés por sus inversiones, en el eventual caso de que las conviertan a colones obtendrán un ingreso adicional.

Las mayores disparidades entre tasas de interés y tipos de cambio se presentaron en el I trimestre 2017 y IV trimestre 2018 con precios por encima del tipo de cambio de proyectado de c5.52 y ¿12.14, respectivamente, lo que favoreció las inversiones en dólares. 
Por otro lado, quienes invirtieron en colones durante el I trimestre 2016, IV trimestre 2016 y II trimestre 2018 , obtuvieron precios de $\$ 22.80$, $\$ 17.59$ y $\$ 29.00$ por debajo del tipo de cambio de proyectado, con la eventualidad de que podían comprar dólares por esos montos por debajo del tipo de cambio de proyectado, y lograr así una mayor ganancia de capital.

Tomando como referencia las tasas de interés netas del sector público costarricense interrelacionadas con las tasas del Tesoro de los Estados Unidos de América se obtuvo un promedio de variación de la diferencia del tipo de cambio, de acuerdo con el efecto Fisher internacional, para el período comprendido del primer trimestre de 2016 al primer trimestre de 2019, un promedio de \$-10.07, nivel máximo de variación correspondiente a \$12.14 en el IV trimestre 2018, y un nivel mínimo de c-29.00 en el II trimestre 2018.

\section{Cálculo del efecto Fisher internacional con tasas de interés netas a diez años}

La misma metodología aplicada a los escenarios anteriores, se aplica el modelo de cálculo del efecto Fisher internacional, ahora a un plazo de diez años y tomando en consideración las tasas de interés en colones en contraposición con las tasas de interés del Tesoro de los Estados Unidos de América.

En la Tabla 7 se presenta la información de las tasas de interés netas respectivas para un plazo de diez años.

Tabla 7

Tasas de Interés netas a diez años. Del IV trimestre 2015 al I trimestre 2016. Por trimestre

\begin{tabular}{lccc} 
Fecha de corte & $\begin{array}{c}\text { Porcentaje de depreciación } \\
\text { trimestral }\end{array}$ & $\begin{array}{c}\text { Tasas de interés netas sector } \\
\text { público CRC }\end{array}$ & $\begin{array}{c}\text { Tasas del Tesoro de } \\
\text { E.U.A. }\end{array}$ \\
\hline IV Trimestre 2015 & $-0,121 \%$ & $5,398 \%$ & $2,270 \%$ \\
I Trimestre 2016 & $-0,485 \%$ & $6,989 \%$ & $1,763 \%$ \\
II Trimestre 2016 & $1,712 \%$ & $6,520 \%$ & $1,471 \%$ \\
III Trimestre 2016 & $2,557 \%$ & $6,591 \%$ & $1,595 \%$ \\
IV Trimestre 2016 & $2,979 \%$ & $6,463 \%$ & $2,476 \%$ \\
I Trimestre 2017 & $1,112 \%$ & $6,398 \%$ & $2,388 \%$ \\
II Trimestre 2017 & $3,345 \%$ & $6,877 \%$ & $2,305 \%$ \\
III Trimestre 2017 & $2,322 \%$ & $8,101 \%$ & $2,334 \%$ \\
IV Trimestre 2017 & $2,042 \%$ & $7,759 \%$ & $2,406 \%$ \\
I Trimestre 2018 & $-0,568 \%$ & $7,825 \%$ & $2,782 \%$ \\
II Trimestre 2018 & $-0,433 \%$ & $8,085 \%$ & $2,861 \%$ \\
III Trimestre 2018 & $2,312 \%$ & $7,825 \%$ & $3,062 \%$ \\
IV Trimestre 2018 & $6,845 \%$ & $9,869 \%$ & $2,685 \%$ \\
I Trimestre 2019 & $-1,535 \%$ & $9,752 \%$ & $2,463 \%$ \\
\hline Fute: & & \\
\hline
\end{tabular}

Fuente: elaboración propia con datos del BCCR. 
En la Tabla 8 se muestra el cálculo del efecto Fisher internacional con aplicación de tasas trimestrales para un plazo de diez años.

Tabla 8

Cálculo del efecto Fisher internacional. Aplicando tasas de interés trimestrales a diez años plazo. Del III trimestre 2016 al I trimestre 2016

\begin{tabular}{lccc}
\hline Fecha de corte & $\begin{array}{c}\text { Tipo de cambio } \\
\text { venta }\end{array}$ & $\begin{array}{c}\text { Proyección efecto Fisher } \\
\text { internacional }\end{array}$ & $\begin{array}{c}\text { Diferencia tipo de cambio venta } \\
\text { y proyectado }\end{array}$ \\
\hline IV Trimestre 2015 & 544,87 & Período base & Período base \\
I Trimestre 2016 & 542,23 & 562,22 & $-19,99$ \\
II Trimestre 2016 & 554,20 & 572,85 & $-18,65$ \\
III Trimestre 2016 & 558,80 & 571,98 & $-13,18$ \\
IV Trimestre 2016 & 561,10 & 571,66 & $-10,56$ \\
I Trimestre 2017 & 567,34 & 566,07 & 1,27 \\
II Trimestre 2017 & 579,87 & 583,08 & $-3,21$ \\
III Trimestre 2017 & 574,13 & 586,18 & $-12,05$ \\
IV Trimestre 2017 & 572,56 & 592,72 & $-20,16$ \\
I Trimestre 2018 & 569,31 & 590,43 & $-21,12$ \\
II Trimestre 2018 & 570,08 & 600,65 & $-30,57$ \\
III Trimestre 2018 & 585,80 & 601,64 & $-15,84$ \\
IV Trimestre 2018 & 611,75 & 599,02 & 12,73 \\
I Trimestre 2019 & 602,36 & 604,22 & $-1,86$ \\
\hline
\end{tabular}

Fuente: elaboración propia con datos del BCCR.

De nuevo se presentan los resultados obtenidos con el mecanismo del modelo de cálculo del efecto Fisher internacional, elaborado por los autores, aplicando, en esta ocasión, una tasa de interés para un plazo de diez años. Los resultados obtenidos en la Tabla 9 significan que cuando la diferencia entre el tipo de cambio de venta es positiva existe un incentivo para invertir en dólares y viceversa para el caso de los colones.

Solamente en el IV trimestre 2018 se refleja una variación significativa por encima del tipo de cambio de venta por un monto de $\$ 12.73$, lo que favoreció las inversiones en dólares.

Por otro lado, aquellos sujetos que invirtieron en colones durante el IV trimestre 2017, I trimestre 2018 y II trimestre 2018, obtuvieron precios de $\$ 20.16$, \21.12 y $\$ 30.57$ por debajo del tipo de cambio en equilibrio, con la eventualidad de que podían comprar dólares por esos montos por debajo del tipo de cambio de equilibrio, obteniendo así una mayor ganancia de capital.

En la Figura. 10 se muestra el comportamiento del tipo de cambio venta y sus proyecciones de acuerdo con las condiciones de mercado presentadas en el momento para tasas de interés a diez años, lo que permite calcular el tipo de cambio esperado según tasas de interés y depreciación proyectada. 
Utilizando el mismo análisis de las figuras anteriores, la Figura 9 muestra el comportamiento del tipo de cambio tanto de venta como el proyectado mediante el efecto Fisher internacional, en donde se establece que cuando la línea celeste, que corresponde al tipo de cambio de venta, se ubica por encima de la línea anaranjada, que corresponde a la proyección del tipo de cambio, quienes han invertido en dólares han recibido un incentivo mayor por sus inversiones.

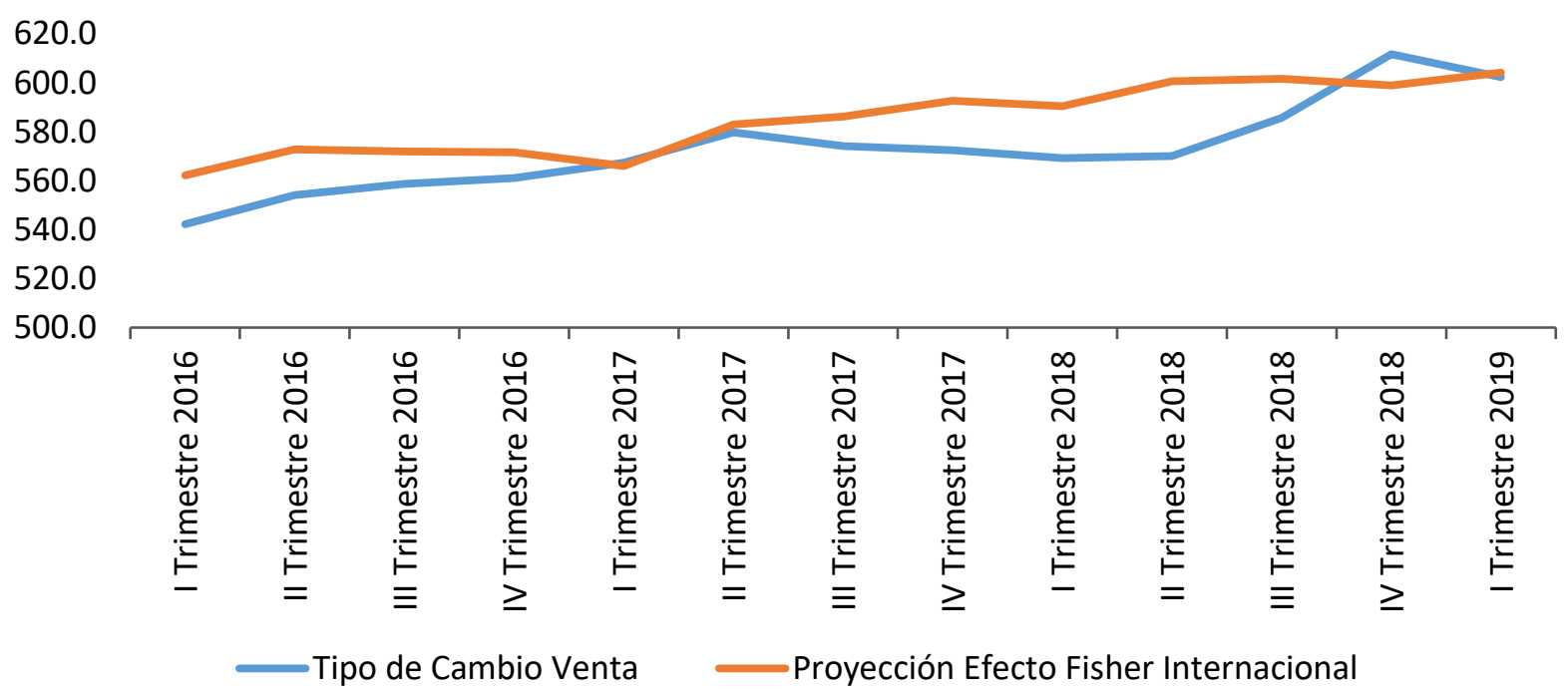

Figura 9. Proyección tipo de cambio y efecto Fisher internacional. Tasas de interés a 10 años del I trimestre 2016 al I trimestre 2019. Fuente: elaboración propia con datos del BCCR.

Tomando como referencia las tasas de interés netas del sector público costarricense interrelacionadas con las tasas del Tesoro de los Estados Unidos de América, ambas a 10 años, se obtuvo un promedio de variación de la diferencia del tipo de cambio, de acuerdo con el efecto Fisher internacional, para el período comprendido entre el primer trimestre de 2016 y el primer trimestre de 2019, un promedio de c-12.43, datos que muestran un nivel máximo de variación correspondiente a $\$ 12.73$ en el IV trimestre 2018 y un nivel mínimo de \$-30.57 para el II trimestre 2018.

\section{Cálculo del efecto Fisher internacional utilizando la tasa básica pasiva y la tasa efectiva en dólares}

En la Tabla 9, el modelo de cálculo del efecto Fisher internacional, elaborado por los autores, toman en cuenta la tasa básica pasiva y la tasa efectiva en dólares, ambos índices calculados y publicados por el BCCR. 
Tabla 9

Tasa básica pasiva y tasa efectiva en dólares. Por trimestre. Del III trimestre 2016 al I trimestre 2016

\begin{tabular}{lccc}
\hline \multicolumn{1}{r}{ Fecha de corte } & $\begin{array}{c}\text { Porcentaje de depreciación } \\
\text { trimestral }\end{array}$ & TBP & TED \\
\hline III Trimestre 2016 & $2,557 \%$ & $4,700 \%$ & $2,010 \%$ \\
IV Trimestre 2016 & $2,979 \%$ & $4,450 \%$ & $2,010 \%$ \\
I Trimestre 2017 & $1,112 \%$ & $4,500 \%$ & $2,160 \%$ \\
II Trimestre 2017 & $3,345 \%$ & $5,700 \%$ & $2,220 \%$ \\
III Trimestre 2017 & $2,322 \%$ & $5,850 \%$ & $2,170 \%$ \\
IV Trimestre 2017 & $2,042 \%$ & $5,950 \%$ & $2,220 \%$ \\
I Trimestre 2018 & $-0,568 \%$ & $2,290 \%$ \\
II Trimestre 2018 & $-0,433 \%$ & $6,000 \%$ & $2,390 \%$ \\
III Trimestre 2018 & $2,312 \%$ & $5,950 \%$ & $2,320 \%$ \\
IV Trimestre 2018 & $6,845 \%$ & $5,900 \%$ & $2,660 \%$ \\
I Trimestre 2019 & $-1,535 \%$ & $6,000 \%$ & $2,770 \%$ \\
\hline
\end{tabular}

Fuente: elaboración propia con datos del BCCR.

En la Tabla 10 se presenta el cálculo del tipo de cambio a futuro "forward" utilizando el modelo del efecto Fisher internacional construido por los autores, se utiliza la tasa básica pasiva y la tasa efectiva en dólares.

Tabla 10

Cálculo del EFI, TBP y TED por trimestre. Del III trimestre 2016 al I trimestre 2019

\begin{tabular}{lccc}
\hline Fecha de corte & $\begin{array}{c}\text { Tipo de cambio } \\
\text { venta }\end{array}$ & $\begin{array}{c}\text { Proyección efecto Fisher } \\
\text { internacional }\end{array}$ & $\begin{array}{c}\text { Diferencia tipo de cambio venta y } \\
\text { proyectado }\end{array}$ \\
\hline III Trimestre 2016 & 558,80 & 561,24 & $-2,44$ \\
IV Trimestre 2016 & 561,10 & 559,24 & 1,86 \\
I Trimestre 2017 & 567,34 & 57,90 & 9,44 \\
II Trimestre 2017 & 579,87 & 573,95 & 5,92 \\
III Trimestre 2017 & 574,13 & 580,20 & $-6,07$ \\
IV Trimestre 2017 & 572,56 & 581,31 & $-8,75$ \\
I Trimestre 2018 & 569,31 & 581,57 & $-12,26$ \\
II Trimestre 2018 & 570,08 & 593,33 & $-23,25$ \\
III Trimestre 2018 & 585,80 & 592,47 & $-6,67$ \\
IV Trimestre 2018 & 611,75 & 592,59 & 19,16 \\
I Trimestre 2019 & 602,36 & 591,19 & 11,17 \\
\hline
\end{tabular}

Fuente: elaboración propia con datos del BCCR.

De la Tabla 10 y de acuerdo con los datos obtenidos de la última columna, cuando la diferencia entre el tipo de cambio de venta y el proyectado según el efecto Fisher internacional es positiva existe un incentivo para invertir en dólares, puesto que, de acuerdo con las variables, el tipo de cambio de colón estaría por encima del de equilibrio. Ello genera una ganancia adicional al ente inversionista en dólares vía tipo de cambio, ya que eventualmente recibiría, aparte de los 
intereses ganados, más colones de los que debería recibir en una condición de equilibrio de tasas de interés o el efecto Fisher internacional.

En la Figura 11 se muestra el comportamiento del tipo de cambio de venta y sus proyecciones de acuerdo con las condiciones de mercado presentadas en el momento, lo que permite calcular el tipo de cambio esperado según tasas de interés y depreciación proyectada. En esta tabla se nota que en la medida en que la línea celeste (tipo de cambio venta) está por encima de la anaranjada (tipo de cambio "forward") todas aquellas inversiones realizadas en dólares han recibido un incentivo mayor por optar por esta moneda, según la misma lógica que se ha venido tratando.

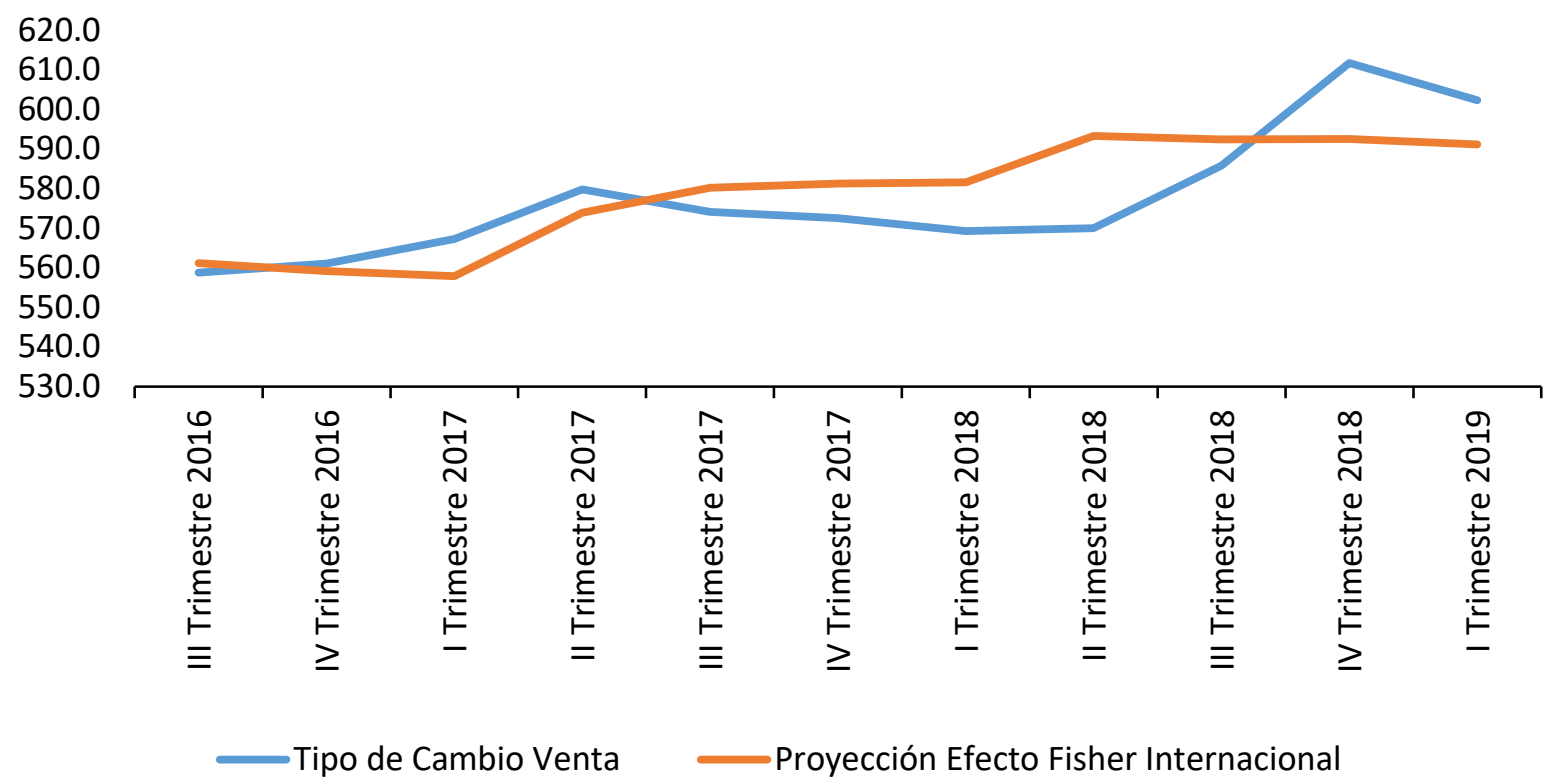

Figura 11. Proyección tipo de cambio, efecto Fisher internacional EFI TBP y TED. Del III trimestre 2016 al I trimestre 2019. Elaboración propia con datos del BCCR.

Las mayores disparidades entre tasas de interés y tipos de cambio se presentaron en el I trimestre 2017, IV trimestre 2018 y I trimestre 2019 con precios por encima del tipo de cambio de equilibrio de $\$ 9.44$, \$19.16 y \$11.17, respectivamente, por encima. Esto favoreció las inversiones en dólares.

Por otro lado, quienes invirtieron en colones durante el IV trimestre 2017, I trimestre 2018 y II trimestre 2018 , obtuvieron precios de $\$ 8.75$, $\$ 12.26$ y $\$ 23.25$ por debajo del tipo de cambio en equilibrio, con la eventualidad de que podían comprar dólares por esos montos por debajo del tipo de cambio de equilibrio, así alcanzan una mayor ganancia de capital. 
Tomando como referencia la tasa básica pasiva interrelacionadas con la tasa efectiva en dólares, ambas calculados por el BCCR, se obtuvo un promedio de variación de la diferencia del tipo de cambio, de acuerdo con el EFI, para el período comprendido entre el tercer trimestre de 2016 y el primer trimestre de 2019 un promedio de $\$$-1.08. Se obtiene en los datos un nivel máximo de variación correspondiente a $\$ 19,16$ en el IV trimestre 2018 y un nivel mínimo de $\$-23.25$ en el II trimestre 2018.

\section{E. Cálculo del EFI utilizando las tasas en dólares y colones transadas en la bolsa nacional de valores de Costa Rica para un plazo de tres años}

Se presenta en la Tabla 11 el último modelo para el cálculo del EFI, elaborado por los autores. Para esta ocasión se toman como parte de las variables las tasas de interés transadas en la Bolsa Nacional de Valores para un plazo de tres años.

Tabla 11

Tasas en dólares y colones transadas a tres años, en la Bolsa Nacional de Valores. Por trimestre. Del IV trimestre 2015 al I trimestre 2016

\begin{tabular}{lccc} 
Fecha de Corte & $\begin{array}{c}\text { Porcentaje de } \\
\text { depreciación trimestral }\end{array}$ & $\begin{array}{c}\text { Rendimiento promedio } \\
\text { colones BNV }\end{array}$ & $\begin{array}{c}\text { Rendimiento promedio } \\
\text { dólares BNV }\end{array}$ \\
\hline IV Trimestre 2015 & $-0,121 \%$ & $6,610 \%$ & $3,750 \%$ \\
I Trimestre 2016 & $-0,485 \%$ & $6,130 \%$ & $3,980 \%$ \\
II Trimestre 2016 & $1,712 \%$ & $5,930 \%$ & $4,200 \%$ \\
III Trimestre 2016 & $2,557 \%$ & $5,990 \%$ & $4,230 \%$ \\
IV Trimestre 2016 & $2,979 \%$ & $5,990 \%$ & $4,230 \%$ \\
I Trimestre 2017 & $1,112 \%$ & $6,410 \%$ & $4,800 \%$ \\
II Trimestre 2017 & $3,345 \%$ & $6,540 \%$ & $4,550 \%$ \\
III Trimestre 2017 & $2,322 \%$ & $7,990 \%$ & $4,560 \%$ \\
IV Trimestre 2017 & $2,042 \%$ & $8,560 \%$ & $4,810 \%$ \\
I Trimestre 2018 & $-0,568 \%$ & $8,750 \%$ & $5,390 \%$ \\
II Trimestre 2018 & $-0,433 \%$ & $8,880 \%$ & $6,060 \%$ \\
III Trimestre 2018 & $2,312 \%$ & $9,110 \%$ & $6,660 \%$ \\
IV Trimestre 2018 & $6,845 \%$ & $9,510 \%$ & $8,330 \%$ \\
I Trimestre 2019 & $-1,535 \%$ & $10,260 \%$ & $8,660 \%$ \\
\hline
\end{tabular}

Fuente: elaboración propia con datos del BCCR.

Para finalizar con esta sección, se presenta la Tabla 12 en donde se realiza el cálculo del tipo de cambio "forward" tomando en consideración el promedio de las tasas de interés de las inversiones que se realizaron tanto en colones como en dólares de forma trimestral, en la Bolsa Nacional de Valores de Costa Rica.

En la Tabla 12 se presenta el cálculo del tipo de cambio a futuro "forward" utilizando para modelo del EFI, construido por los autores, y las tasas de interés promedio de transacciones a tres años en la Bolsa Nacional de Valores por trimestre. 
Tabla 12

Cálculo del EFI, tasas de interés promedio de transacciones a tres años en la Bolsa Nacional de Valores por trimestre. Del IV trimestre 2015 al I trimestre 2019

\begin{tabular}{lccc}
\hline Fecha de corte & Tipo de cambio venta & $\begin{array}{c}\text { Proyección efecto Fisher } \\
\text { internacional }\end{array}$ & $\begin{array}{c}\text { Diferencia tipo de cambio venta } \\
\text { y proyectado }\end{array}$ \\
\hline IV Trimestre 2015 & 544,87 & Período base & Período base \\
I Trimestre 2016 & 542,23 & 560,57 & $-18,34$ \\
II Trimestre 2016 & 554,20 & 556,14 & $-1,94$ \\
III Trimestre 2016 & 558,80 & 553,92 & 4,88 \\
IV Trimestre 2016 & 561,10 & 554,07 & 7,03 \\
I Trimestre 2017 & 567,34 & 554,07 & 13,27 \\
II Trimestre 2017 & 579,87 & 569,72 & 10,15 \\
III Trimestre 2017 & 574,13 & 571,78 & 2,35 \\
IV Trimestre 2017 & 572,56 & 579,51 & $-6,95$ \\
I Trimestre 2018 & 569,31 & 581,18 & $-11,87$ \\
II Trimestre 2018 & 570,08 & 590,81 & $-20,73$ \\
III Trimestre 2018 & 585,80 & 587,78 & $-1,98$ \\
IV Trimestre 2018 & 611,75 & 585,71 & 26,04 \\
I Trimestre 2019 & 602,36 & 578,80 & 23,56 \\
\hline
\end{tabular}

Fuente: elaboración propia con datos del BCCR.

Con la aplicación el modelo de cálculo del efecto Fisher internacional para las tasas de interés en colones y dólares a un plazo de tres años, ofrecidas por la Bolsa Nacional de Valores, se obtiene de la Tabla 12 un comportamiento del tipo de cambio "forward" tal, que cuando la diferencia entre el tipo de cambio de venta y el proyectado es positiva, el ente inversionista en dólares obtendrá una ganancia, producto no solo de los intereses recibidos por la inversión, sino también por la conversión de esos dólares a colones; puesto que el tipo de cambio futuro sería mayor al tipo de cambio inmediato; situación contraria sucede, si la diferencia es negativa.

En la Figura 12 se muestra el comportamiento del tipo de cambio venta y sus proyecciones de acuerdo con las condiciones de mercado presentadas en el momento, lo que permite calcular el tipo de cambio esperado según tasas de interés y depreciación proyectada. La línea anaranjada corresponde al tipo de cambio de venta del Banco Centra de Costa Rica y la línea celeste al tipo de cambio "forward" calculado con el modelo desarrollado por los autores.

Las mayores disparidades entre tasas de interés y tipos de cambio se presentaron en el I trimestre 2017, IV trimestre 2018 y I trimestre 2019 con precios por encima del tipo de cambio de equilibrio de \$13.27, ¿26.04 y \23.56, respectivamente, por encima, ello favoreció las inversiones en dólares.

Por otro lado, aquellos grupos que invirtieron en colones durante el I trimestre 2016, I trimestre 2018 y II trimestre 2018 obtuvieron precios de \$18.34, \$11.87 y $\$ 20.73$ por debajo del tipo de 
cambio en equilibrio, con la eventualidad de que podían comprar dólares por esos montos por debajo del tipo de cambio de equilibrio y ganar, así, mayor capital.

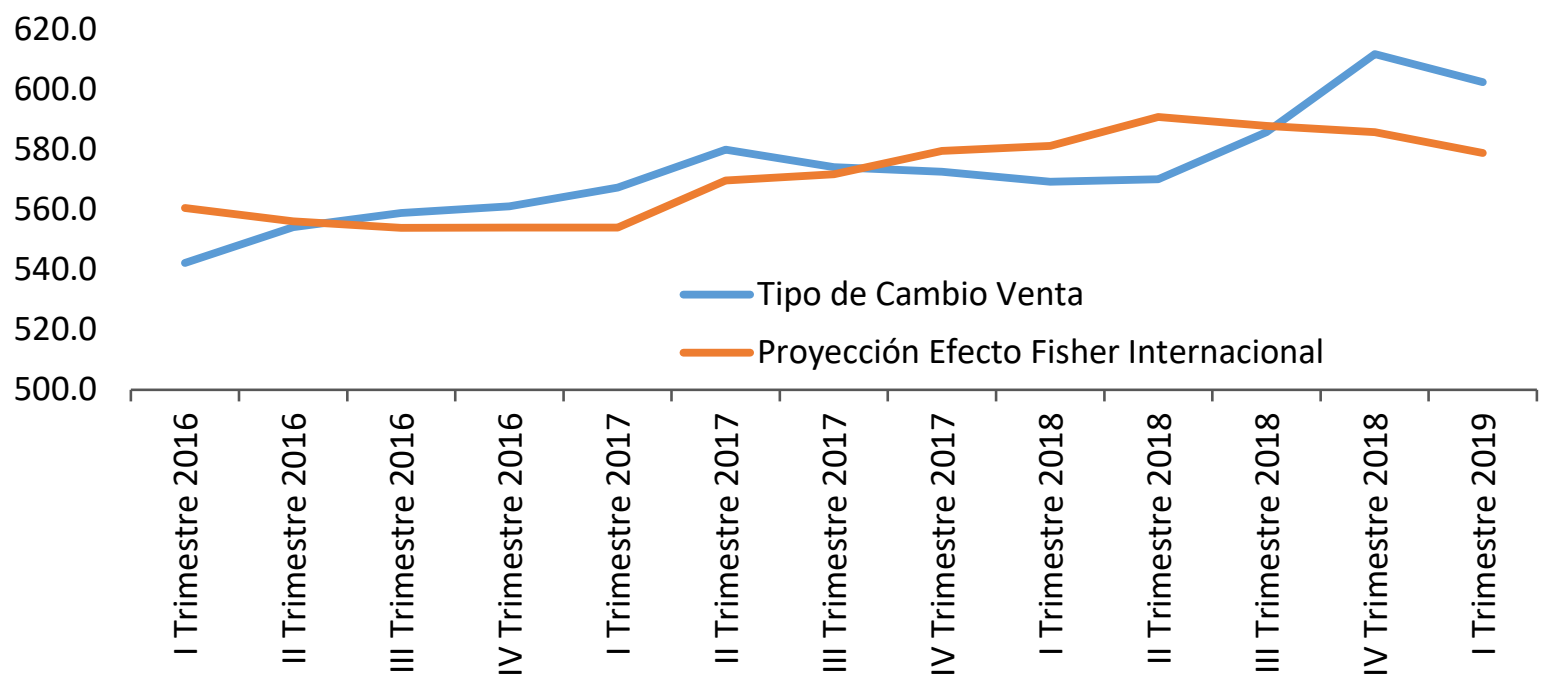

Figura 12. Proyección tipo de cambio y EFI, tasas de interés promedio a tres años de transacciones en la Bolsa Nacional de Valores por trimestre. Del I trimestre 2016 al I trimestre 2019. Elaboración propia con datos del BCCR.

Tomando como referencia las tasas de interés de las inversiones que se realizaron tanto en colones como en dólares por trimestre, para una plazo de tres meses, en la Bolsa Nacional de Valores se obtuvo un promedio de variación de la diferencia del tipo de cambio, de acuerdo con el efecto Fisher internacional, para el período comprendido del primer trimestre de 2016 al primer trimestre de 2019 de $\$ 1.96$, con datos de un nivel máximo de variación correspondiente a \26.04 para el IV trimestre 2018 y un nivel mínimo de \$-20.73 para el segundo trimestre 2018. Realizado el análisis de todos los modelos de cálculo del efecto Fisher internacional, no se puede obviar que, en los meses de diciembre 2018 y enero 2019, la calificación de riesgo de Costa Rica para moneda extranjera se redujo por parte de tres calificadoras de riesgo internacional, lo que convierte las inversiones realizadas por parte de inversionistas del exterior, en menos atractivas y con un riesgo mayor. La cronología de esta evaluación fue:

> 5 de diciembre: la calificadora Moody's disminuyó la calificación de B1 a Ba2.

> 21 de diciembre: Standard \& Poor's redujo la calificación de BB- a B+.

$>15$ enero 2019: se presentó un descenso en la calificación por parte de Fitch Rating, calificación que pasó de "BB" a "B+" descendiendo así dos peldaños.

De lo anterior se deduce que a nivel internacional y tomando en consideración los diferentes parámetros de estas calificadoras, el descenso se le atribuye principalmente a la situación de coyuntura fiscal por la que está pasando el país y a las necesidades de recursos por parte del gobierno, pero a nivel interno incide en el grado de riesgo de las inversiones financieras. 


\section{Análisis de resultados}

A partir del cálculo del efecto Fischer internacional, es posible esbozar algunos elementos relevantes para invertir en dólares, mediante el comportamiento del tipo de cambio de venta durante el período en estudio (I trimestre 2016 al I trimestre 2019).

Como se recordará, si la diferencia entre el tipo de cambio de venta (o inmediato) y el tipo de cambio proyectado (forward) es negativa, se obtiene una ganancia de capital si se convierten estos dólares a colones; caso contrario, si el resultado es negativo. Para sintetizar el comportamiento del tipo de cambio durante el período en estudio y poder identificar estos elementos relevantes para la toma de decisiones de inversión en dólares, se elaboró la tabla 13. Se observa que, durante el período en estudio, las diferencias han sido mayoritariamente negativas con 29 observaciones contra 10 observaciones positivas. Esto representa un $74 \%$ de resultados negativos contra un $26 \%$ de positivos, por lo que tenemos que las instancias tomadoras de decisión que optaron por realizar este tipo de inversión obtuvieron una ganancia de capital.

Otro elemento importante consiste en que cuando los entes inversionistas observan, en el mercado, que el tipo de cambio de venta o inmediato muestra una disminución en su valor absoluto, comienzan su proceso de convertir sus posesiones en moneda extranjera (USD) a moneda nacional (CRC), como se evidencia entre los I trimestre de 2017, el IV trimestre de 2018 y I trimestre del 2019.

Tabla 13

Comparación de la según plazo de tasas de interés relacionado con cambio absoluto en tipo de cambio venta diferencia tipo de cambio venta y proyectado. Del I trimestre 2016 al I trimestre 2019

\begin{tabular}{lccccc} 
Trimestres & Dos años & Cinco años & Diez años & $\begin{array}{c}\text { Tipo de cambio } \\
\text { venta }\end{array}$ & $\begin{array}{c}\text { Cambio absoluto TC } \\
\text { venta }\end{array}$ \\
\hline I T 2016 & $-19,49$ & $-22,8$ & $-19,99$ & 542,23 & $-2,64$ \\
II T 2016 & $-6,28$ & $-13,71$ & $-18,65$ & 554,2 & 11,97 \\
III T 2016 & $-1,71$ & $-8,97$ & $-13,18$ & 558,8 & 4,6 \\
IV T 2016 & 0,25 & $-6,34$ & $-10,56$ & 561,1 & 2,3 \\
I T 2017 & 9,04 & 5,52 & 1,27 & 567,34 & 6,24 \\
II T 2017 & 4,23 & 0,12 & $-3,21$ & 579,87 & 12,53 \\
III T 2017 & $-13,85$ & $-13,48$ & $-12,05$ & 574,13 & $-5,74$ \\
IV T 2017 & $-15,52$ & $-17,59$ & $-20,16$ & 572,56 & $-1,57$ \\
I T 2018 & $-15,99$ & $-20,41$ & $-21,12$ & 569,31 & $-3,25$ \\
II T 2018 & $-25,62$ & $-29,00$ & $-30,57$ & 570,08 & 0,77 \\
III T 2018 & $-9,97$ & $-14,49$ & $-15,84$ & 585,8 & 15,72 \\
IV T 2018 & 18,30 & 12,14 & 12,73 & 611,75 & 25,95 \\
I T 2019 & 8,38 & $-1,86$ & $-10,26$ & 602,36 & $-9,39$ \\
\hline
\end{tabular}

Fuente: elaboración propia con datos del BCCR. 
En la Tabla 14 se muestra el plazo que ha generado más ganancia de capital, a partir de la consideración del plazo de tasas de interés y la clasificación de los resultados de la diferencia entre el tipo de cambio inmediato y futuro en positivos y negativos.

Tabla 14

Clasificación de resultados del efecto Fisher internacional según plazo de tasas de interés netas. I trimestre 2016 - I trimestre 2019

\begin{tabular}{ccccccc}
\hline $\begin{array}{c}\text { Observación/ } \\
\text { Plazo }\end{array}$ & \multicolumn{2}{c}{ 2 años } & \multicolumn{2}{c}{$\mathbf{5}$ años } & \multicolumn{2}{c}{$\mathbf{1 0}$ años } \\
Absoluta & Relativa & Absoluta & Relativa & Absoluta & Relativa \\
\hline Positivas & 5 & 0,38 & 3 & 0,23 & 2 & 0,15 \\
Negativas & 8 & 0,62 & 10 & 0,77 & 11 & 0,85 \\
Total & 13 & $100 \%$ & 13 & $100 \%$ & 13 & $100 \%$ \\
\hline
\end{tabular}

Fuente: elaboración propia con datos del BCCR.

En conclusión, se puede evidenciar que el plazo de tasa de interés más probable para obtener una ganancia de capital para una inversión en dólares durante el período de estudio es el de 10 años.

\section{Conclusiones}

El cálculo del efecto Fisher internacional tiene una importancia fundamental, no solo para la enseñanza de las finanzas internacionales, sino también para la toma de decisiones de inversión en dólares, sobre todo en sistemas económicos - como es el caso de Costa Rica- donde el tipo de cambio se fija por un régimen cambiario flexible, ya que dicha inversión puede generar ganancias de capital para las instancias tomadoras de decisiones en esta opción de inversión.

Del período de estudio, del I trimestre de 2016 al I trimestre de 2019, se evidencia que la opción de invertir en dólares, en la mayoría de las veces, generó ganancia de capital para las inversiones en esta moneda.

También se pudo evidenciar que quienes invierten en dólares se guían por el cambio absoluto en el valor del tipo de cambio en aras de lograr una ganancia de capital.

A partir del modelo utilizado, la inversión de dólares tiene una mayor probabilidad de generar una ganancia de capital en los plazos de tasas de interés a largo plazo.

Con los datos obtenidos y la construcción del modelo, se puede concluir que en la actualidad existe un incentivo para quienes realicen inversiones en moneda extranjera, especialmente por los siguientes dos aspectos: dada la baja de la calificación de riesgo internacional para Costa Rica, se tiene que crear un atractivo, incentivo o premio por invertir en colones y es, necesariamente,

Carlos Leonardo Arguedas Campos y Gustavo Vallejo Esquivel

\section{(c) (i) (2)}

Revista Economía y Sociedad by Universidad Nacional is licensed under a CreativeCommons Reconocimiento-NoComercial- 
a través de la aplicación de tasas de interés favorables que compensen el riego, de forma tal de que se cumpla la regla financiera de que a mayor riesgo mayor tasa de interés.

El gobierno de Costa Rica también busca hacer atractiva la deuda interna, por lo que procede también a ofrecer tasas de captación atractivas con respecto al colón.

En cuanto al comportamiento de la tasa básica pasiva, tasa efectiva en dólares, el tipo de cambio y su impacto en la población costarricense, se puede recomendar que al igual que el resto de los indicadores económicos del BCCR, la tasa básica pasiva no puede verse como un indicador aislado, ya que este interactúa como un engrane más de la política económica del BCCR, que dentro de sus objetivos institucionales contempla la "estabilidad, competitividad y eficiencia de intermediación financiera", y como objetivo estratégico "promover un sistema financiero estable y competitivo", por lo que de este indicador dependen directamente los ahorros y los créditos que realicen los individuos y las empresas a lo largo del territorio nacional.

El caso de las inversiones en dólares, pese a mantener un nivel bajo y estable en cuanto a tasas de interés (y ha sucedido con los colones), desde abril de 2017 ha mostrado un alza en las transacciones de esta moneda, producto del alza del dólar, por especulación y la situación fiscal del país.

Siguiendo la idea anterior, tanto para los entes deudores como para los inversionistas se vislumbra una estabilidad en cuanto a tipo de cambio y tasas de interés en dólares se refiere, en tanto el BCCR cuente con reservas suficientes para intervenir el mercado, tal y como lo ha venido realizando en momentos de estrés cambiario, como el que se presentó en el segundo semestre del 2017. 


\section{Referencias}

Banco Central de Costa Rica. (1995). Ley Orgánica del Banco Central. N. ${ }^{\circ} 7558$. Recuperado de http://www.pgrweb.go.cr/scij/Busqueda/Normativa/Normas/nrm texto completo.aspx? param1=NRTC\&nValor1=1\&nValor2=40928

Banco Central de Costa Rica. (2019). Indicadores: Tasa básica pasiva, información diaria. Recuperado de http://indicadoreseconomicos.bccr.fi.cr/indicadoreseconomicos/Cuadros/frmVerCatCuad ro.aspx?idioma $=1 \&$ CodCuadro $=\% 2017$

Banco Central de Costa Rica. (2019). Indicadores: Tipo cambio de compra y de venta del dólar de los Estados Unidos de América, referencia Banco Central de Costa Rica. Recuperado de http://indicadoreseconomicos.bccr.fi.cr/indicadoreseconomicos/Cuadros/frmVerCatCuad ro.aspx? idioma $=1 \&$ CodCuadro $=\% 20400$

Contraloría General de la República. (2018). Resultado de servicios de transportes y carreteras. Recuperado de https://cgrfiles.cgr.go.cr/publico/docsweb/enpsp-2018/transporte.html

Ministerio Economía, Industria y Comercio. (2019). Estudios de tarjetas. Recuperado de https://www.meic.go.cr/meic/web/284/estudios/tarjetas-de-credito-y-debito/tarjetascredito-debito.php 